\title{
Cluster observations of flux rope structures in the near-tail
}

\author{
P. D. Henderson ${ }^{1}$, C. J. Owen ${ }^{1}$, I. V. Alexeev ${ }^{1}$, J. Slavin ${ }^{2}$, A. N. Fazakerley ${ }^{1}$, E. Lucek ${ }^{3}$, and H. Rème ${ }^{4}$ \\ ${ }^{1}$ Mullard Space Science Laboratory, University College London, Holmbury St. Mary, Dorking, RH5 6NT, UK \\ ${ }^{2}$ Laboratory for Extraterrestrial Physics, NASA GSFC, Greenbelt, MD, 20771, USA \\ ${ }^{3}$ Space and Atmospheric Physics, Imperial College London, SW7 2BZ, UK \\ ${ }^{4}$ Centre d'Etude Spatiale des Rayonnements, Toulouse, France
}

Received: 26 August 2005 - Revised: 17 December 2005 - Accepted: 10 January 2006 - Published: 23 March 2006

\begin{abstract}
An investigation of the 2003 Cluster tail season has revealed small flux ropes in the near-tail plasma sheet of Earth. These flux ropes manifest themselves as a bipolar magnetic field signature (usually predominantly in the Zcomponent) associated with a strong transient peak in one or more of the other components (usually the Y-component). These signatures are interpreted as the passage of a cylindrical magnetic structure with a strong axial magnetic field over the spacecraft position. On the 2 October 2003 all four Cluster spacecraft observed a flux rope in the plasma sheet at $\mathrm{X}$ $(\mathrm{GSM}) \sim-17 R_{E}$. The flux rope was travelling Earthward and duskward at $\sim 160 \mathrm{kms}^{-1}$, as determined from multispacecraft timing. This is consistent with the observed souththen-north bipolar $\mathrm{B}_{\mathrm{Z}}$ signature and corresponds to a size of $\sim 0.3 R_{E}$ (a lower estimate, measuring between the inflection points of the bipolar signature). The axis direction, determined from multi-spacecraft timing and the direction of the strong core field, was close to the intermediate variance direction of the magnetic field. The current inside the flux rope, determined from the curlometer technique, was predominantly parallel to the magnetic field. However, throughout the flux rope, but more significant in the outer sections, a non-zero component of current perpendicular to the magnetic field existed. This shows that the flux rope was not in a "constant $\alpha$ " force-free configuration, i.e. the magnetic force, $\boldsymbol{J} \times \boldsymbol{B}$ was also non-zero. In the variance frame of the magnetic field, the components of $\boldsymbol{J} \times \boldsymbol{B}$ suggest that the magnetic pressure force was acting to expand the flux rope, i.e. directed away from the centre of the flux rope, whereas the smaller magnetic tension force was acting to compress the flux rope. The plasma pressure is reduced inside the flux rope. A simple estimate of the total force acting on the flux rope from the magnetic forces and surrounding plasma suggests that the flux rope was experiencing an expansive total force.
\end{abstract}

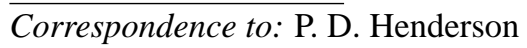

(pdh@mssl.ucl.ac.uk)
On 13 August 2003 all four Cluster spacecraft observed a flux rope at $\mathrm{X}(\mathrm{GSM}) \sim-18 R_{E}$. This flux rope was travelling tailward at $200 \mathrm{kms}^{-1}$, consistent with the observed north-then-south bipolar $\mathrm{B}_{\mathrm{Z}}$ signature. The bipolar signature corresponds to a size of $\sim 0.3 R_{E}$ (lower estimate). In this case, the axis, determined from multi-spacecraft timing and the direction of the strong core field, was directed close to the maximum variance direction of the magnetic field. The current had components both parallel and perpendicular to the magnetic field, and $\boldsymbol{J} \times \boldsymbol{B}$ was again larger in the outer sections of the flux rope than in the centre. This flux rope was also under expansive magnetic pressure forces from $\boldsymbol{J} \times \boldsymbol{B}$, i.e. directed away from the centre of the flux rope, and had a reduced plasma pressure inside the flux rope. A simple total force calculation suggests that this flux rope was experiencing a large expansive total force.

The observations of a larger $\boldsymbol{J} \times \boldsymbol{B}$ signature in the outer sections of the flux ropes when compared to the centre may be explained if the flux ropes are observed at an intermediate stage of their evolution after creation by reconnection at multiple X lines near the Cluster apogee. It is suggested that these flux ropes are in the process of relaxing towards the force-free like configuration often observed further down the tail. The centre of the flux ropes may contain older reconnected flux at a later evolutionary stage and may therefore be more force-free.

Keywords. Magnetospheric physics (Magnetotail; Plasma sheet; Storms and substorms)

\section{Introduction}

Flux ropes have been interpreted as evidence for multiple Xline reconnection (MXR) in the near-tail associated with substorms (e.g. Elphic et al., 1986; Slavin et al., 2003a; Slavin et al., 2003b; Deng et al., 2004). The study of the formation and evolution of flux ropes, and therefore MXR, is important in learning more about the development of the current

Published by Copernicus GmbH on behalf of the European Geosciences Union. 


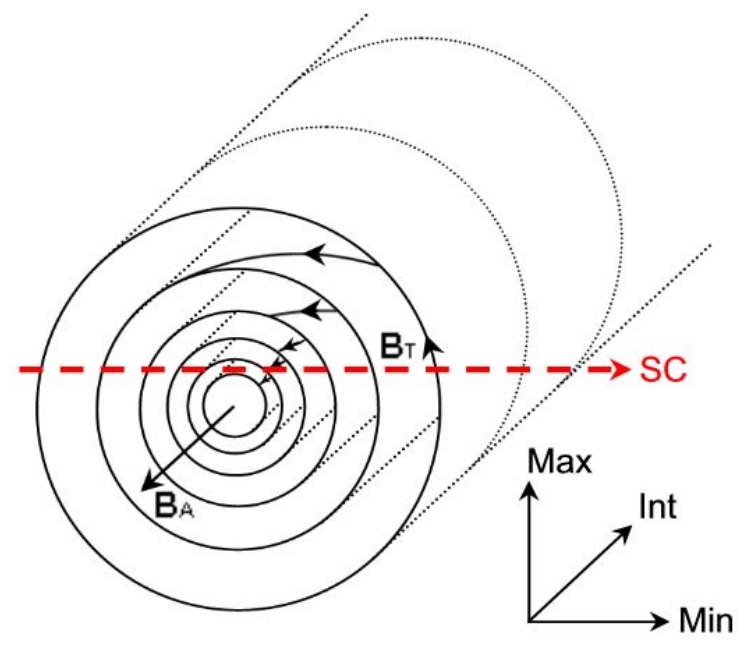

Fig. 1. The topology of a force-free helical flux rope. The strong axial and tangential magnetic field directions are marked. In the centre of the rope $\mathrm{B}_{\mathrm{T}}$ is zero, increasing to a maximum at the edge, whilst $\mathrm{B}_{\mathrm{A}}$ is maximal at the centre, falling to a minimum at the edge of the flux rope. A cartoon spacecraft trajectory is marked, along with the variance coordinate system that would arise from a constant $\alpha$ force-free flux rope.

sheet during the substorm process. In MXR, instead of creating one single $\mathrm{X}$-line in the tail, the conditions required for reconnection can be satisfied in numerous places, creating a number of X-lines. Given an IMF $B_{Y}$ component which penetrates into the tail (Hughes and Sibeck, 1987), flux ropes can be created between such X-lines. As Schindler (1974) first noted, one X-line will generally reconnect plasma sheet field lines more rapidly than others. Reconnection at this X-line will be first to propagate out to open field lines in the lobe, thus becoming the single dominant magnetotail $\mathrm{X}$-line. This $\mathrm{X}$-line then produces Alfvénic jets in the Earthward and tailward directions. The newly-formed flux ropes between the remaining $\mathrm{X}$-lines will be embedded in these flows and thus move away from the point at which they were created. In this simplest picture, the cores of the flux ropes are nominally directed across the tail (i.e. mainly in the GSM Y-direction) and the flux ropes travel towards or away from the Earth (i.e. mainly in the GSM X-direction), depending on which side of the dominant $\mathrm{X}$-line they are located.

The gross, large scale properties of magnetotail flux ropes have been studied with the use of single spacecraft techniques (Elphic et al., 1986; Moldwin and Hughes, 1991; Slavin et al., 1995; Slavin et al., 2003a), but only recently have their small-scale properties been investigated with Cluster (Slavin et al., 2003b; Zong et al., 2004; Eastwood et al., 2005). Observations of these flux ropes are characterised by a bipolar $\mathrm{B}_{\mathrm{Z}}$ signature, caused by the magnetic structure moving past the spacecraft, and often show a large increase in the magnitude of $\boldsymbol{B}$ caused by a strong core field. Events with a south-then-north (north-then-south) signature are seen to move Earthward (tailward), and are indeed usually embedded in fast plasma flows (Slavin et al., 2003a).

The simplest magnetic flux rope model is the "force-free" flux rope. This model represents the minimum energy state for helical magnetic field lines, and could therefore represent the cores of well developed, fully evolved flux ropes observed in the deep tail (e.g. Moldwin and Hughes, 1991; Slavin et al., 1995). A popular mathematical representation of a force-free flux rope is known as the "constant $\alpha$ " solution (e.g. Lepping et al., 1990). In this class of force-free flux rope, $\boldsymbol{J}=\alpha \boldsymbol{B}$, where $\boldsymbol{J}$ is the current density vector. The topology of the magnetic field is a nested set of helical magnetic field lines ranging from a relatively weak tangential field on the outer boundary of the flux rope to a strong core axial field in the centre. This magnetic field topology is represented schematically in Fig. 1. At all places in the flux rope the magnetic force, $\boldsymbol{J} \times \boldsymbol{B}=0$, since the current vector is everywhere parallel to the magnetic field vector. In this model, as $|\boldsymbol{J}|$ is proportional to $|\boldsymbol{B}|$, the current in the centre of the rope is mainly axial and strongly peaked.

Surveys of flux ropes in the tail have been performed previously (Elphic et al., 1986; Slavin et al., 1995; Slavin et al., 2003a). Slavin et al. (1995) reported evidence for the existence of tailward moving plasmoids with force-free flux rope topologies in the distant tail $\left(\mathrm{X} \sim-230 R_{E}\right)$. The observations of a strong core field or HFR (High Field Region) with typical scale sizes of $\sim 5$ to $10 R_{E}$ led the authors to suggest that a force-free core was embedded in a non force-free plasmoid. Surveys of Geotail data (Slavin et al., 2003a) reported many $\sim 2$ to $5 R_{E}$ diameter flux ropes between 15 and $30 R_{E}$ down the tail. A large proportion of these flux ropes was found to fit well with the model outlined above and to be fairly cylindrical in shape. This led to the conclusion that the $\boldsymbol{J} \times \boldsymbol{B}$ forces were small, i.e. many of these flux ropes were in a force-free configuration. This paper reported that a similar proportion of flux ropes were observed moving tailwards as Earthwards. Furthermore, Cluster observations of travelling compression regions (Slavin et al., 2005), a probable signature of the passage of flux ropes in the lobes, show that at very near-tail distances (i.e. $\mathrm{X}>\sim-20 R_{E}$ ) $80 \%$ of flux ropes are traveling Earthward. Slavin et al. (2003b) reported on a flux rope observed by Cluster in the 2001 tail season, where all four spacecraft sampled the structure directly. This was unexpected as the separation of the Cluster spacecraft was almost comparable to the size of the flux rope $\left(\sim 1 R_{E}\right)$. Slavin et al. (2003b) concluded that this flux rope was not in a force-free configuration as the perpendicular currents were larger than the parallel currents for the first half of the flux rope encounter, although they noted a good agreement with the magnetic field topology of the type illustrated in Fig. 1.

In this paper, data from the Cluster mission is used along with applications of multi-spacecraft analysis methods to investigate flux ropes in the near-tail region of the magnetosphere of Earth. Multi-spacecraft timing and the calculation of an average curl of the magnetometer data is used in order 
to probe the internal structure of two such flux ropes. For the first time the magnetic forces are computed throughout flux ropes.

The analysis techniques used in this paper are described in the next section. Flux ropes in the 2003 Cluster tail season are discussed in Sect. 3. Two case studies of flux ropes observed by Cluster are detailed in Sects. 4 and 5. The results of the case studies and their implications are discussed in Sect. 6.

\section{Analysis techniques}

Previous single spacecraft studies of flux ropes have used minimum variance analysis (Sonnerup and Cahill, 1967) in an attempt to determine the orientation of the structures (Elphic et al., 1986; Slavin et al., 2003a; Slavin et al., 2003b; Xiao et al., 2004). Minimum variance analysis is frequently used as a means to define a natural flux rope coordinate system. For the constant $\alpha$ force-free model, it can be shown that a variance analysis on the magnetic field components will give an intermediate variance direction which corresponds to the axis of the flux rope (Xiao et al., 2004). Moreover, analysis of magnetic field data from a spacecraft following a trajectory directly through the middle of a constant $\alpha$ forcefree flux rope which is invariant along its axis will find the minimum variance direction to be along the spacecraft trajectory. The field in this direction will have zero magnetic field and therefore variance of this field. The maximum variance direction is directed along a tangent to the cylindrical outer magnetic field line and the magnetic field in this direction will exhibit the bipolar signature seen in the study of flux ropes. In Fig. 1 a coordinate system is sketched that would arise from a minimum variance analysis of the magnetic field data from a spacecraft following the marked trajectory (dashed line) through the structure. In this structure, the magnetic field along the intermediate variance direction will peak at closest approach to the centre of the flux rope and at the zero crossing of the bipolar signature in the maximum variance direction. For trajectories that do not pass directly through the middle of the flux rope, the magnetic field in the intermediate and maximum variance directions will have smaller amplitude, while the magnetic field along the minimum variance direction will have non-zero variance. It can therefore be seen that, as the trajectory moves away from the centre of the flux rope, the magnetic field in the intermediate and minimum variance directions begin to look similar. A good separation between all three of the eigenvalues of the minimum variance analysis is therefore needed to accurately determine the flux rope orientation using this method. It is important to note, however, that these variance analysis results may also arise from other flux rope models (both forcefree and non-force-free). Indeed, more complicated structures may return different orientations of the variance analysis system with respect to the flux rope axis. For example, the intermediate variance direction may not necessarily correspond to the axis in a non-force-free flux rope if there exists a very strong core field. Thus the minimum variance analysis may not always accurately determine a "flux rope frame" (Moldwin and Hughes, 1991; Slavin et al., 2003b). Further diagnostics are thus needed if to establish accurately the structure of such flux ropes.

Cluster is a four spacecraft mission whose orbit is such that for three months of any year the apogee is in the Earth's magnetotail at a distance $\sim 20 R_{E}$. In 2003, the typical separation of the Cluster spacecraft was only $200 \mathrm{~km}$, a separation useful for the determination of the properties of small-scale structures in the near-tail region. A unique set of tools and techniques is made possible with the multi-spacecraft nature of Cluster. By simultaneously measuring the magnetic field at four different spacecraft, the average curl of the magnetic field can be computed using difference equations. An average current through the Cluster tetrahedron can therefore be derived. This method is called the "curlometer" (Robert et al., 1998; Dunlop et al., 2002). The curlometer technique assumes that there is a linear field gradient between the spacecraft, implying that over the spacecraft tetrahedron the current is constant. The accuracy of the derived current can only be determined by noting the deviation of the measured current from a model current. However, with no a priori knowledge of the field to be observed, the quality of the result must be determined in other ways. As the divergence of the field should be zero, the calculated divergence of the magnetic field is a good quality indicator to use in place of the error. There is no direct one-to-one correlation between the error and the divergence of the magnetic field but it can be used in place of the error for simple current structures such as flux ropes. Many caveats to this statement exist and are detailed in Robert et al. (1998) and Dunlop et al. (2002). The error in the curlometer current is mainly dependent on the spatial gradients of the current structure and the spatial sampling of the spacecraft. The assumption that the current is constant over the tetrahedron is most likely to be valid when the spacecraft separation is small, as in the 2003 tail season. The best spatial sampling comes when the Cluster spacecraft are in a regular tetrahedral formation. The spatial sampling is characterised with the use of the elongation (E) and planarity (P) parameters. These parameters, ranging from 0 to 1 , define a suite of spacecraft configurations. The optimum configuration for most multi-spacecraft techniques is the "pseudo-sphere", characterised by $\sqrt{E^{2}+P^{2}}$ below $\sim 0.4$. Robert et al. (1998) and Dunlop et al. (2003) conclude that, if the spacecraft separation is small compared to the current structure being observed and the tetrahedron is regular (taken here as $\sqrt{E^{2}+P^{2}}$ being small), the divergence is a good indicator of the quality of the result in place of the error. By dividing the divergence by the curl of the magnetic field a quantity is produced that is here identified with the relative error, $\xi$, in the curlometer result: 


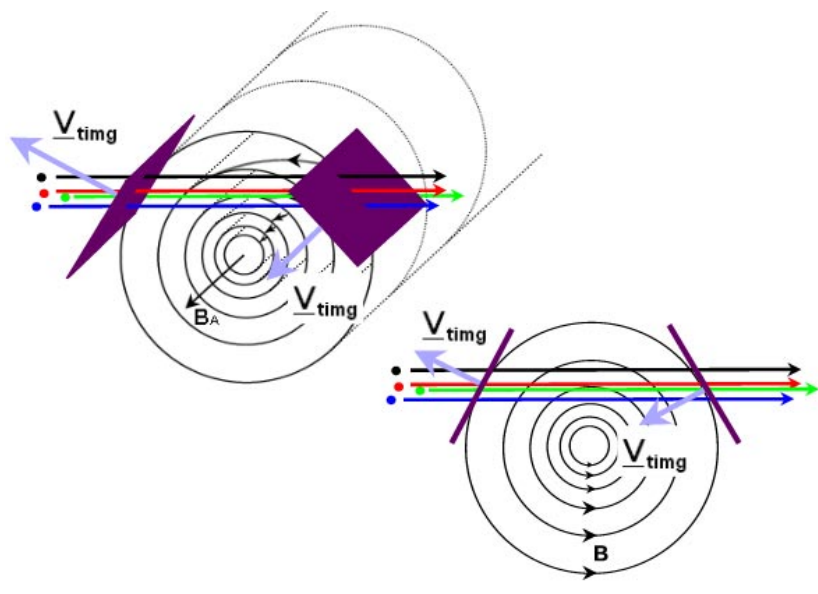

Fig. 2. Cartoon of constant flux surfaces. By measuring the time lag between observations of constant flux surfaces at each Cluster spacecraft, the marked surfaces and velocities can be constructed.

$\xi=\frac{\nabla . \boldsymbol{B}}{|\nabla \times \boldsymbol{B}|}$

This quantity was used in Slavin et al. (2003b) and Xiao et al. (2004) and mentioned in Eastwood et al. (2002) as a good indicator of the quality of the curlometer results in the contexts of both two near-tail flux rope studies and of a heliospheric flux rope study.

With knowledge of the curl of the magnetic field and the gradient of the magnitude of the magnetic field, the magnetic forces inside the flux ropes can be investigated.

$\boldsymbol{J} \times \boldsymbol{B} \equiv \frac{(\nabla \times \boldsymbol{B}) \times \boldsymbol{B}}{\mu_{0}}=\frac{-1}{\mu_{0}}|\boldsymbol{B}|(\nabla|\boldsymbol{B}|)+\frac{1}{\mu_{0}}(\boldsymbol{B} . \nabla) \boldsymbol{B}$

Here $\frac{-1}{\mu_{0}}|\boldsymbol{B}|(\nabla|\boldsymbol{B}|)$ is usually referred to as the "magnetic pressure" force, whereas $\frac{1}{\mu_{0}}(\boldsymbol{B} . \nabla) \boldsymbol{B}$ is referred to as the "magnetic tension" force. In this interpretation of magnetic force, the two terms arise from an over or under-density of magnetic flux relative to surroundings and curved magnetic field lines respectively.

The four spacecraft nature of Cluster can also used for multi-spacecraft timing, also known as four spacecraft timing (4SCT). By noting certain corresponding "events" in the four spacecraft data sets occurring at different times, it is possible to derive the velocity of a surface through the tetrahedron. This analysis is valid when the separation of the spacecraft is small enough that the disturbance being investigated can be assumed to be planar and moving at a constant velocity. In this study of flux ropes, the time at which each spacecraft observes a particular value of $|\boldsymbol{B}|$ (constant flux surface) near or shortly after (before) the time of the first (second) inflection point of the bipolar signature is noted. These instances are associated with the times at which each Cluster spacecraft observes the outer boundary of the flux rope. Figure 2 shows a cartoon of the two surfaces that would be resolved if this method was applied to a force-free flux rope. A planar surface can be constructed to locally approximate the curved flux rope surface. The velocity of the constructed surface along its normal, $\underline{V}_{\text {timg }}$, can be computed. Levels of constant $|\boldsymbol{B}|$, as opposed to $\mathrm{B}_{\mathrm{Z}}$ or the magnetic field in any variance direction, are frame independent and remove any uncertainty arising from the choice of frame. For an axially invariant flux rope, the cross product of the inbound and outbound $\underline{V}_{\text {timg }}$ vectors can be used to define a plane whose normal represents the axis of the flux rope, i.e.

$\underline{\hat{\boldsymbol{V}}}_{\text {timg }}{ }^{1} \times \underline{\hat{\boldsymbol{V}}}_{\text {timg }}{ }^{2} \sim \underline{\hat{\boldsymbol{e}}}_{\text {axis }}$

Where $\underline{\hat{\boldsymbol{e}}}_{\text {axis }}$ is a unit vector along the axis. If the flux rope is in a force-free configuration, the intermediate variance direction should correspond with the axis determined from 4SCT (Xiao et al., 2004).

$\underline{\hat{\boldsymbol{e}}}_{\text {axis }} \sim \underline{\hat{\boldsymbol{e}}}_{\text {intermediate }}$

Where $\underline{\boldsymbol{e}}_{\text {intermediate }}$ is the unit vector along the intermediate variance direction. Consequently, if the structure is close to cylindrical, Eq. (3) gives an independent estimate of the axis of the structure, regardless of whether the structure is in a force-free configuration or not, and can act as an independent test of the applicability of the minimum variance analysis. If minimum variance fails to give a framework in which the structure of a flux rope may be easily determined, as could happen in a non-force-free flux rope, this method could be used to find an axial direction. This method can be used for flux ropes observed in the 2003 tail season, provided the scale size of the flux rope is significantly larger than the spacecraft separation, $200 \mathrm{~km}\left(0.03 R_{E}\right)$.

\section{Flux ropes in the 2003 cluster tail season}

An investigation of the 2003 Cluster small separation tail season has been undertaken in an attempt to find flux ropes in the plasma sheet using the Cluster Fluxgate Magnetometer (FGM) (Balogh et al., 2001) and the Plasma Electron And Current Experiment (PEACE) (Johnstone et al., 1997) instruments. Periods when Cluster was close to apogee in the tail between early August and late October were investigated. Spin averaged data ( $\sim 4$-s resolution) were used in the initial stages of the investigation, with high resolution magnetic field data being employed to study events in further detail. A threshold on $|\boldsymbol{B}|(30 \mathrm{nT})$ and a clear signature of plasma sheet electrons in PEACE was used to ensure that only the times when Cluster was in the plasma sheet were investigated. The periods that satisfied these tests were then manually surveyed. The criterion for the identification of a flux rope was a clear north-then-south or south-then-north bipolar signature (i.e. a rotation of the magnetic field component between two points) associated with a clear increase in $|\boldsymbol{B}|$ (to eliminate magnetic loop events, which generally exhibit a bipolar signature with no, or little increase in $|\boldsymbol{B}|$ ). 


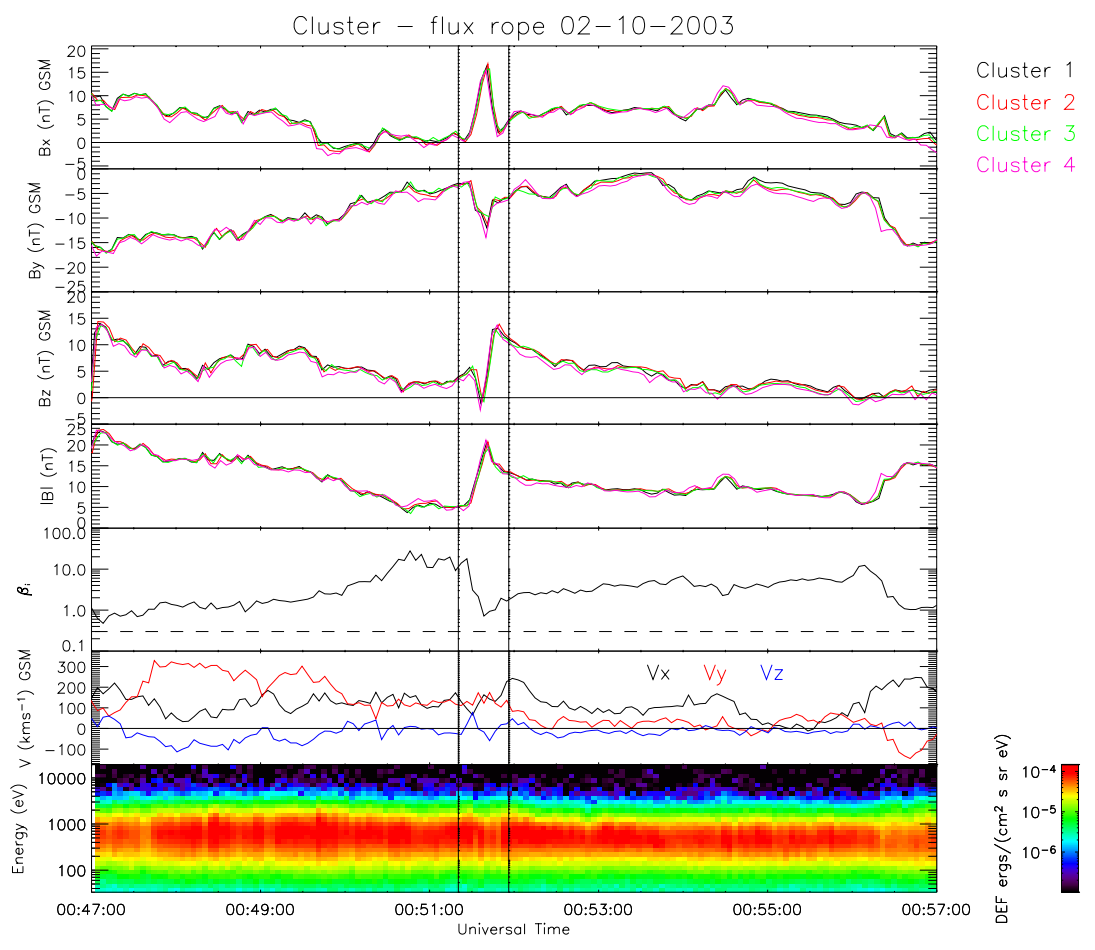

Fig. 3. Cluster observations of 2 October 2003. Panels 1 to 4 show components of $\boldsymbol{B}$ and $|\boldsymbol{B}|$ from each spacecraft (Cluster $1-$ black, Cluster 2 - red, Cluster 3 - green, Cluster 4 - magenta). Panel 5 shows plasma $\beta_{\mathrm{i}}$, derived from Cluster 1 ion moments. $\beta_{\mathrm{i}}=0.3$ is marked, a value of $\beta_{\mathrm{i}}$ above this is indicative of plasma sheet conditions. Panel 6 shows velocities from CIS (Cluster $1, \mathrm{~V}_{\mathrm{x}}$ black, $\mathrm{V}_{\mathrm{y}}$ red, $\mathrm{V}_{\mathrm{z}}$ blue). Panel 7 contains a spectrogram of the direction-averaged differential energy flux for electrons in the energy range $30 \mathrm{eV}-30 \mathrm{KeV}$ recorded by the PEACE instrument on Cluster 4. The flux rope event is marked between two black lines.

Ten flux rope candidates were found in the data that satisfied all of the above criteria. However, two such events will be presented in this paper. These events were selected due to their clear core fields and bipolar signatures.

\section{Case study 1, 2 October 2003}

On 2 October 2003 at approximately 01:00:00 UT, the Cluster spacecraft were located $\sim(-17,8,-3) R_{E}$ (GSM coordinates will be used throughout this paper unless otherwise stated). Figure 3 shows three components of the magnetic field from all spacecraft (Cluster 1 - black, Cluster 2 - red, Cluster 3 - green, Cluster 4 - magenta) and their magnitudes for the period 00:47:00 to 00:57:00 UT. Panel 5 shows the ion plasma beta $\left(\beta_{\mathrm{i}}\right)$ from the Cluster Ion Spectrometry (CIS) experiment (Reme et al., 2001) on Cluster 1 , with $\beta_{\mathrm{i}}=0.3$ marked by a dotted line. Values of $\beta_{\mathrm{i}}$ above this value are indicative of plasma sheet conditions. A difference is noted in the ion density determined from on-board moments and ground moments. The density generated from ground moments (12-s time resolution) is here higher than that generated from on-board moments (4-s time resolution). In order to generate confidence in the plasma pressure measurements, the plasma pressure was "calibrated" by assuming that total pressure was constant across the tail for the period close to the flux rope encounter. This process assumed that all plasma pressure measurements were modified by a constant multiplicative factor. For ground moments this factor was close to 1.0 , giving confidence in the ground moments. For onboard moments this factor was close to 1.35. After taking this factor into account it was found that the plasma pressure determined from on-board moments and ground moments agree well (not shown). These "corrected" ion pressure onboard moments (4-s time resolution) are used throughout this study and are used here in the calculation of $\beta_{\mathrm{i}}$. Panel 6 shows the components of plasma velocity from CIS $\left(\mathrm{V}_{\mathrm{x}}\right.$ black, $\mathrm{V}_{\mathrm{y}}$ red, $\mathrm{V}_{\mathrm{z}}$ blue) on Cluster 1 . These velocity moments are determined on board the spacecraft and agree well with lower time-resolution velocity moments determined by ground analysis (not shown) and are therefore not modified. The bottom panel contains a spectrogram of the directionaveraged differential energy flux for electrons in the energy range $30 \mathrm{eV}-30 \mathrm{keV}$ recorded by the PEACE instrument on Cluster 4.

The PEACE energy spectrogram shows a large differential energy flux of $\sim 1 \mathrm{keV}$ electrons. Together with magnetic field observations showing $\mathrm{B}_{\mathrm{x}}(\sim 0 \mathrm{nT}),|\boldsymbol{B}|(\sim 5 \mathrm{nT})$ and $\beta_{\mathrm{i}}$ ranging between $\sim 1$ to 10 , this confirms that the 


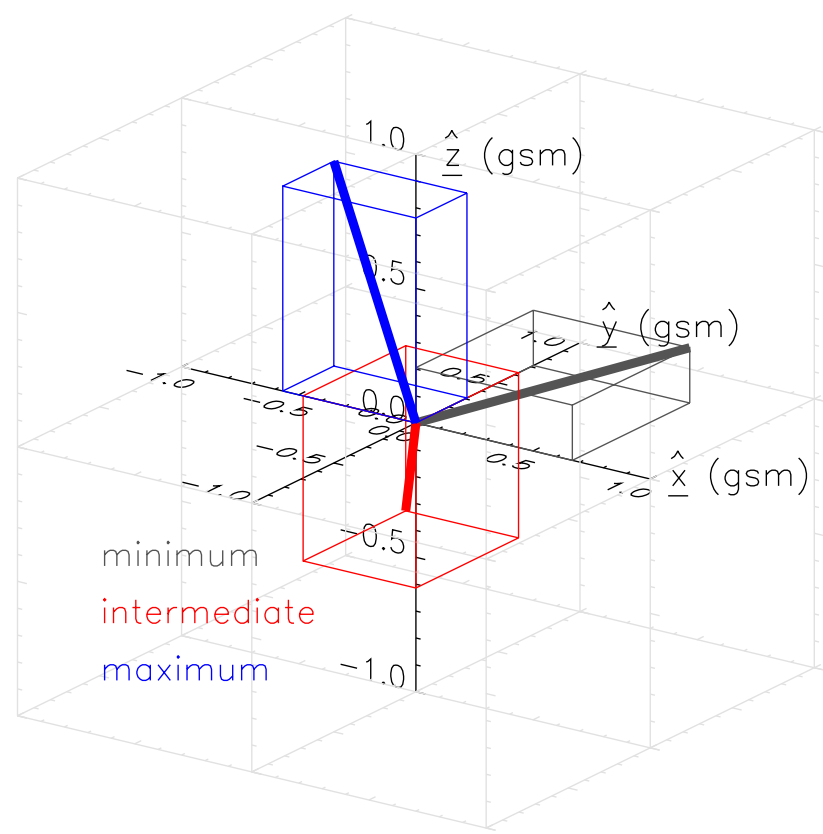

Fig. 4. A 3-D view of the variance system in GSM unit space from Cluster 1 for the 2 October 2003 event. The minimum variance direction is coloured black, intermediate variance direction is coloured red and maximum variance direction is coloured blue. Thin tracer lines are plotted giving the projections onto the GSM unit axes as a guide to the direction of the vectors.

Cluster spacecraft were in the plasma sheet throughout the period shown. At approximately 00:52:00 UT a large spike in $|\boldsymbol{B}|$ can be seen (to $\sim 20 \mathrm{nT}$ ), along with a sharp increase in $\mathrm{B}_{\mathrm{x}}$ (to $\sim 15 \mathrm{nT}$ ) and $\mathrm{B}_{\mathrm{y}}$ (from $\sim-5 \mathrm{nT}$ to $\sim-15 \mathrm{nT}$ ). A bipolar south-then-north $\mathrm{B}_{\mathrm{Z}}$ signature is also evident. The $\mathrm{AE}$ index for this time (not shown) indicates that the magnetosphere was in the recovery phase of a large substorm. The souththen-north bipolar signature suggests a flux rope moving in the Earthward direction. CIS ion velocity shows that this flux rope was embedded in a fast flow in the $+\mathrm{X}\left(\sim 150 \mathrm{kms}^{-1}\right)$ $+\mathrm{Y}\left(\sim 150 \mathrm{kms}^{-1}\right)$ direction, i.e. Earthwards and duskwards. The deflection in $\mathrm{B}_{\mathrm{x}}$ (positive) and $\mathrm{B}_{\mathrm{y}}$ (negative) indicates a strong core field in the $+\mathrm{X}$ and $-\mathrm{Y}$-direction. The bipolar signature is not symmetric which is the result of a core field contribution in $B_{Z}$ (positive), i.e. the axis is aligned ( $+X,-Y$, $+\mathrm{Z}$ ) (see below).

A minimum variance analysis was carried out on high resolution FGM data. Figure 4 shows the orientation of the variance directions determined from Cluster 1 with respect to the GSM axes. This figure is a 3-D view of the GSM system (unit vectors used) where the minimum variance direction is coloured black, intermediate variance direction is coloured red and maximum variance direction is coloured blue. Thin tracer lines show the projections of each vector onto the unit GSM axes. The direction of maximum variance is determined with a larger confidence than are the

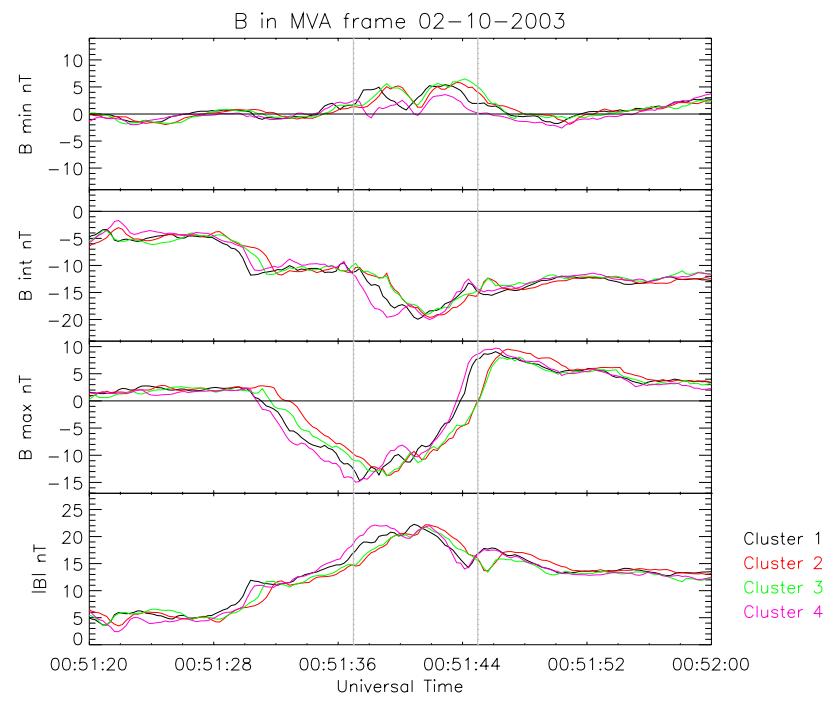

Fig. 5. Magnetic field observations from 2 October event transformed into the variance frame of Cluster 1; the magnetic field in the minimum, intermediate and maximum variance directions (Fig. 4) and the magnitude from all spacecraft. Cluster colours are in the same format as those in Fig. 3. The approximate boundaries of the flux ropes, located at the inflection points, are marked by grey lines.

directions of minimum and intermediate variance since the minimum-intermediate eigenvalue ratio is smaller than the intermediate-maximum eigenvalue ratio for all spacecraft. The frame with the largest eigenvalue separation was selected, which in this case was that returned from analysis of Cluster 1 data, where the minimum-intermediate-maximum eigenvalue ratios were 1:3.2:17.9. The minimum variance direction is mostly in the $+\mathrm{X}+\mathrm{Y}$ direction. The intermediate variance direction is highly inclined to the $\mathrm{XY}$ plane. If, as would be expected for a constant $\alpha$ force-free flux rope, this direction corresponds to the flux rope axis, the axis would be in the $(-\mathrm{X},+\mathrm{Y},-\mathrm{Z})$ direction consistent with the opposite direction of the core field in the magnetic field measurements. The core field should therefore be negative along the intermediate variance direction.

The magnetic field observations are plotted in the minimum variance frame in Fig. 5. This figure shows four panels; the magnetic field in the minimum, intermediate and maximum variance directions and the magnitude from all spacecraft, with spacecraft colours in the same format as those in Fig. 3. Grey lines mark the approximate boundaries of the flux rope, identified in this paper as the locations of the inflection points of the bipolar signature and the central core magnetic field. The magnetic fields in both the minimum and intermediate variance directions are peaked. The larger negative peak in the intermediate variance direction represents the core field. The bipolar signature appears in the maximum variance direction (a rotation of the magnetic field between $\sim-15 \mathrm{nT}$ and $9 \mathrm{nT}$ for Cluster 1). This is consistent 


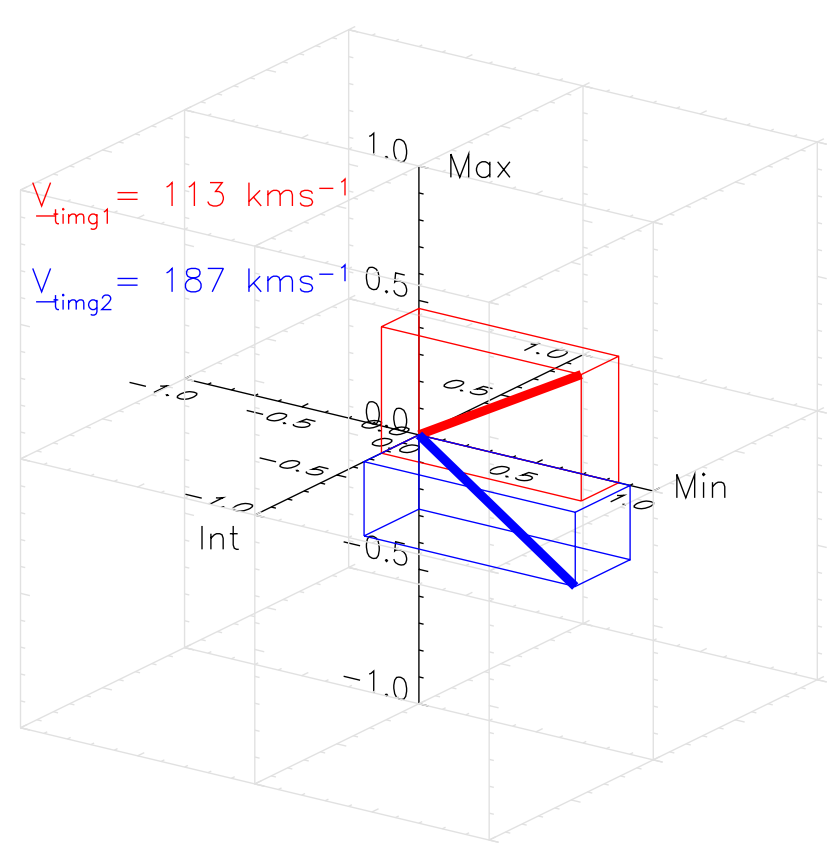

Fig. 6. A $3-D$ view of the variance frame with $\underline{V}_{\text {timg }}$ vectors produced from constant flux surfaces on 2 October event. The directions "Min", "Int" and "Max" correspond to the minimum, intermediate and maximum variance directions plotted in GSM unit space in Fig. 4.

with expectations from the simple constant $\alpha$ force-free flux rope. The enhancement in $|\boldsymbol{B}|$ suggests a spacecraft closest approach time of 00:51:41 UT. As described above, 4SCT provides a test of the minimum variance analysis results. This analysis was performed on levels of constant magnetic field magnitude for both the inbound (20 nT) and outbound $(18 \mathrm{nT})$ parts of the encounter and the $\underline{V}_{\text {timg }}$ vectors produced can be seen in Fig. 6 in the variance frame of Cluster 1. This figure is a 3-D view of the variance analysis axes (unit vectors used). The first $\underline{V}_{\text {timg }}$ is coloured red, the second coloured blue. Thin tracer lines show the projections of each vector onto the unit variance axes.

It can be seen that both inbound and outbound timing vectors lie close to the minimum-maximum variance plane and define a plane whose normal is close to the intermediate variance direction (see Eq. 3). This result suggests that the intermediate variance direction is indeed close to the axis of the flux rope and that the variance analysis is returning a system that is consistent with a natural "flux rope frame".

4SCT can also be used to compute a velocity of the flux rope over the spacecraft. Figure 7 shows a schematic of the velocities involved. In this figure the surface being investigated is moving along $\underline{\boldsymbol{V}}$, while the normal to the surface is $\underline{\hat{n}}$. The velocity $\underline{V}_{\text {timg }}$ returned by 4 SCT is the velocity of the surface along the normal, $\left|\underline{\boldsymbol{V}}_{\mathrm{timg}}\right|=\underline{\boldsymbol{V}} \cdot \underline{\hat{n}}$, where $\underline{\boldsymbol{V}}$ is the velocity of the flux rope. If the minimum variance direction can be assumed to be along or close to the trajectory, then

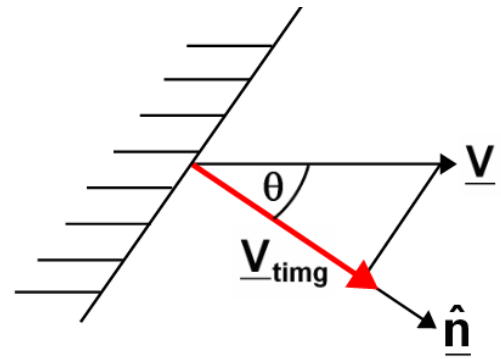

Fig. 7. Schematic of the velocities computed in 4SCT. The velocity $\underline{V}_{\text {timg }}$ returned by $4 \mathrm{SCT}$ is the velocity of the surface along the normal.

the velocity $\underline{\boldsymbol{V}}$ along this direction can be calculated by $|\underline{\boldsymbol{V}}|=$ $\left|\underline{\boldsymbol{V}}_{\text {timg }}\right| / \cos (\theta)$ where $\theta$ is the angle between $\underline{\boldsymbol{V}}$ and $\underline{\boldsymbol{V}}$ timg. This was calculated on both inbound $\left(\sim 130 \mathrm{kms}^{-1}\right)$ and outbound $\left(\sim 190 \mathrm{kms}^{-1}\right)$ vectors and an average taken. A resultant velocity of $160 \mathrm{kms}^{-1}$ corresponds to a size of $\sim 0.3 R_{E}$ (taking the time between the inflection points of $\sim 10 \mathrm{~s}$ ) for this flux rope. This is a lower estimate of the diameter of the flux rope, $\sim 0.3 R_{E}$ representing the distance between the boundaries of the flux rope at this particular impact parameter, which is here defined as the relative distance from the centre of the flux rope. The velocity of the rope determined by $4 \mathrm{SCT}$ is so directed that the rope is travelling Earthward, in agreement with the south-then-north polarity of the $\mathrm{B}_{Z}$ signature and CIS ion velocities. CIS ion velocities rotated into the minimum variance frame (not shown) give a velocity along the minimum variance direction of $\sim 190 \mathrm{kms}^{-1}$ in approximate agreement with the velocities derived from 4SCT, with smaller velocities along the intermediate and maximum variance directions of $\sim 40 \mathrm{kms}^{-1}$ and $\sim-60 \mathrm{kms}^{-1}$, respectively.

Note that 4SCT assumes that the size of the flux rope is large enough that the surface encountered can be considered planar. As the size of the tetrahedron is $\sim 0.03 R_{E}$, ten times smaller than the inferred size of the rope, this assumption should be valid in this case.

The curlometer technique described above was applied to this period of data and the results are shown in Fig. 8. Panel 1 shows the current parallel to the magnetic field (black) and the magnitude of the current perpendicular to the magnetic field (red). Note that the barycentric magnetic field is used; i.e. the predicted magnetic field at the geometric centroid of the Cluster tetrahedron. Panel 2 shows the current projected into the minimum (black), intermediate (red) and maximum (blue) variance directions of the magnetic field from Cluster 1 (Fig. 4) respectively. Panel 3 shows the magnitude of the current. Panel 4 shows the relative error in the curlometer current, $\xi$ (Eq. 1). For this event $\mathrm{E}=0.21, \mathrm{P}=0.30$ giving a $\sqrt{E^{2}+P^{2}}$ of 0.37 . The tetrahedron is therefore a good example of a pseudo-sphere and $\xi$, identified as the relative error, should give a good measure of the quality of the curlometer 


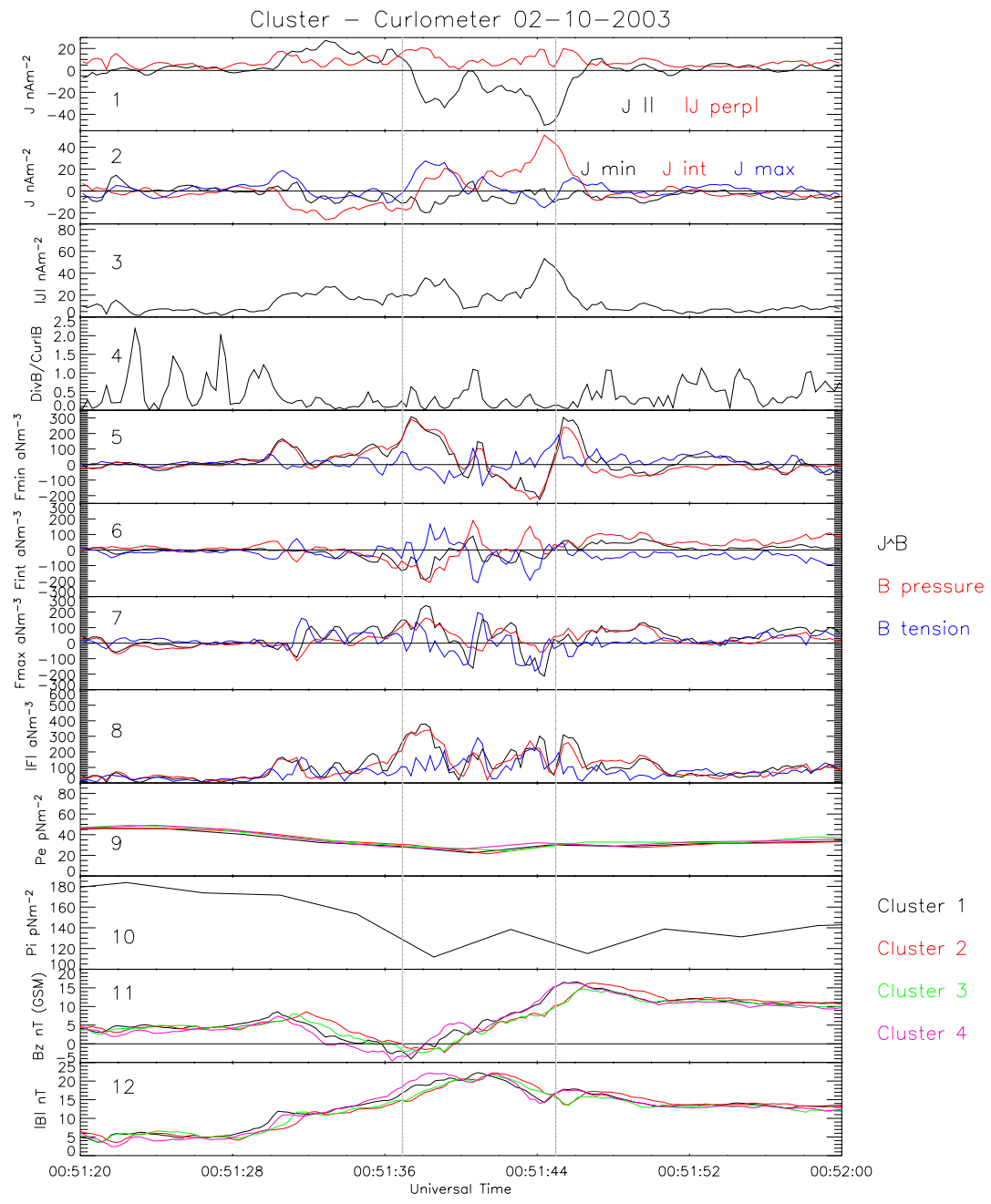

Fig. 8. Cluster curlometer observations of 2 October 2003. Panel 1 shows the current parallel (black) and the magnitude of the current perpendicular (red) to the magnetic field. Panel 2 shows the current projected into the minimum (black), intermediate (red) and maximum (blue) variance directions respectively. Panels 3 and 4 show the magnitude of the current and the fractional error in the curlometer result respectively. Panels 5 to 7 show $\boldsymbol{J} \times \boldsymbol{B}$ (black), magnetic pressure (red) and magnetic tension (blue) projected onto the minimum, intermediate and maximum variance directions of Cluster 1 respectively. Panel 8 shows $|\boldsymbol{J} \times \boldsymbol{B}|$ and the magnitudes of the magnetic pressure and tension forces. Panels 9 and 10 show the electron and ion pressure respectively, with the final two panels showing the $\mathrm{B}_{\mathrm{Z}}$ and $|\boldsymbol{B}|$ observations displaying the bipolar signature and increase in $|\boldsymbol{B}|$.

result. Panels 5 to 8 show the magnetic force (black, barycentric magnetic field used), the magnetic pressure force (red, see Eq. 2) and the magnetic tension force (blue, calculated from $\left.\boldsymbol{J} \times \boldsymbol{B}-\frac{-1}{\mu_{0}}|\boldsymbol{B}|(\nabla|\boldsymbol{B}|)\right)$ transformed into the minimum, intermediate and maximum variance directions of the magnetic field of Cluster 1, and their magnitudes. Panels 9 and 10 show the electron and ion pressure derived from PEACE and CIS data respectively. The final two panels again show the $\mathrm{B}_{\mathrm{Z}}$ component and $|\boldsymbol{B}|$ from each spacecraft for reference.

Panels 1 and 3 show that there are three large current enhancements around the time of the flux rope encounter and that the first enhancement (00:51:33 UT) is largely directed parallel, the second two enhancements (00:51:38 and 00:51:44 UT) are directed anti-parallel (but with a small perpendicular component) to the magnetic field direction. As the boundary of the flux rope is identified as being between the two inflection points of the bipolar signature $(\sim$ grey vertical lines), the first current enhancement (parallel to the magnetic field) is outside the flux rope by this definition (i.e. just Earthward of the flux rope), while the second two current enhancements are inside the structure. Also, the current is smaller in the centre of the flux rope than near the edges. It can be seen from Panel 2 that the smaller first current enhancement inside the flux rope (00:51:38 UT) is in the intermediate (red) and maximum (blue) variance directions, whereas the larger second current enhancement (00:51:44 UT) is in the intermediate variance direction (red). This current would therefore seem to be mainly axially 
directed, but not located in the centre of the flux rope. The smaller currents in the maximum variance direction (blue) show a reversal during the flux rope encounter, first positive along the maximum variance direction (00:51:38 UT) changing to negative (00:51:44 UT). $\xi$ (Panel 4) is small, being generally less than 0.5 over the whole of the flux rope, apart from one small section close to the centre (00:51:41 UT) where the error becomes $\sim 1$. In this region the current is small, meaning that a large $\xi$ will have an insignificant result on the overall current. Panel 5, the magnetic force along the minimum variance direction (assumed to be the trajectory), shows two direction reversals, or a tripolar signature. The force along this direction is first positive (00:51:37 UT), turning negative (00:51:44 UT), then quickly turning positive again very close to the trailing edge of the flux rope (00:51:46 UT). The direction reversal over the flux rope (i.e. between vertical grey lines) suggests that the magnetic force is acting to expand the flux rope, i.e. acting away from the flux rope centre, consistent with the magnetic pressure dominance of the total magnetic force. The total magnetic force along the axis (Panel 6) is generally smaller than that of any other component. The magnetic pressure and tension forces generally have opposite signs along all directions, but are not balanced. Note that a force-free flux rope will have an exact magnetic pressure-tension force balance in the absence of plasma pressure. The electron and ion pressures (Panels 9 and 10) are reduced inside the flux rope.

In order to compute the total sum of forces acting on this flux rope, the spatial gradient of plasma pressure must be compared to the magnetic forces. The gradient in the pressure cannot here be calculated because CIS aboard Cluster 2 is not operational and the pressure gradient structure cannot be compared to the small scale magnetic force structure because of the low time resolution of the plasma instruments. In order to make an estimate of the total force acting on the flux rope the change in plasma pressure recorded at a point just before (or after) and inside the flux rope is compared to the magnetic pressure (calculated by $|\boldsymbol{B}|^{2} / 2 \mu_{0}$ ) change measured on the same spacecraft over the same time range. Assuming most of the magnetic force comes from the magnetic pressure force (as it does in this case) a useful estimate of the balance of forces (both plasma and magnetic) can be made across the flux rope. Over the first half of the flux rope (Cluster 1 ion and magnetic pressure evaluated at 00:51:31 UT and 00:51:39 UT with the Cluster 1 electron pressure evaluated at 00:51:33 and 00:51:41 UT) a simple comparison shows that the change in magnetic pressure is a factor of $\sim 1.4$ larger than that of the plasma pressure. Over the second half of the flux rope (ion and magnetic pressure evaluated at 00:51:39 UT and 00:51:51 UT, electron pressure evaluated at 00:51:41 and 00:51:53 UT) the comparison again suggests that the change in magnetic pressure is larger than that of the plasma pressure, but this time by a factor of $\sim 2.3$. This would seem to suggest that the flux rope is experiencing an expansive total force.
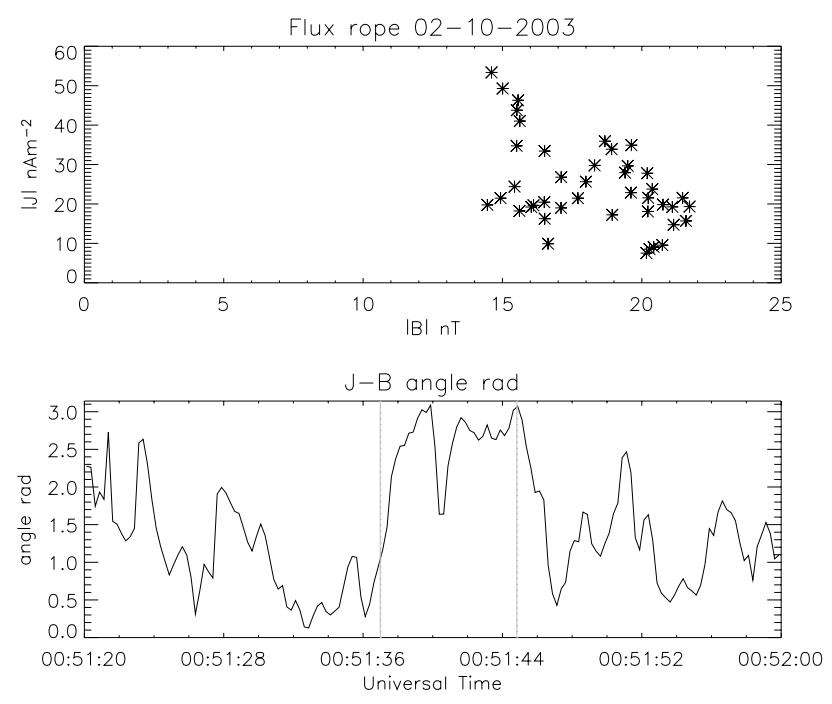

Fig. 9. $|\boldsymbol{J}|$ versus $|\boldsymbol{B}|$ and the angle (in radians) between the magnetic field and current density vectors for the 2 October 2003 flux rope. For a constant $\alpha$ force-free flux rope $|\boldsymbol{J}|$ would be proportional to $|\boldsymbol{B}|$ and the magnetic field and current density vectors would be parallel.

Figure 9 shows a scatter plot of the magnitudes of the current density and magnetic field, along with the angle (in radians) between the current density and magnetic field vectors. In a constant $\alpha$ force-free flux rope $|\boldsymbol{J}|$ is proportional to $|\boldsymbol{B}|$, and the current density and magnetic field vectors would be parallel. $|\boldsymbol{J}|$ versus $|\boldsymbol{B}|$ for this event yields no clear proportionality and there exists a non-constant non-zero angle between the current density and magnetic field vectors. These results are inconsistent with the flux rope being in a constant $\alpha$ force-free configuration.

If the magnetic force can be assumed to be acting only radially, finding the direction along which the magnetic force is minimal can give another estimate of the axial direction. A minimum variance analysis carried out on $\boldsymbol{J} \times \boldsymbol{B}$ gives the minimum variance direction to be $(-0.759,0.613,-0.219)$ with a minimum-intermediate eigenvalue ratio of 5.0. This direction makes an angle of $28^{\circ}$ to the intermediate variance direction of the magnetic field, the assumed axis: $(-0.481$, $0.625,-0.614)$. The maximum variance direction of the current density is $(-0.026,-0.442,0.897)$ with an intermediatemaximum eigenvalue ratio of 3.0. This makes an angle of $35^{\circ}$ to the assumed axis and confirms that the current is not simply axial.

\section{Case study 2, 13 August 2003}

On the 13 August at approximately 03:00:00 UT, all four Cluster spacecraft were located at $\sim(-18,-7,0) R_{E}$. Figure 10 shows the components of the magnetic field, CIS $\beta_{\mathrm{i}}$ (using "corrected" on-board density moments) and 


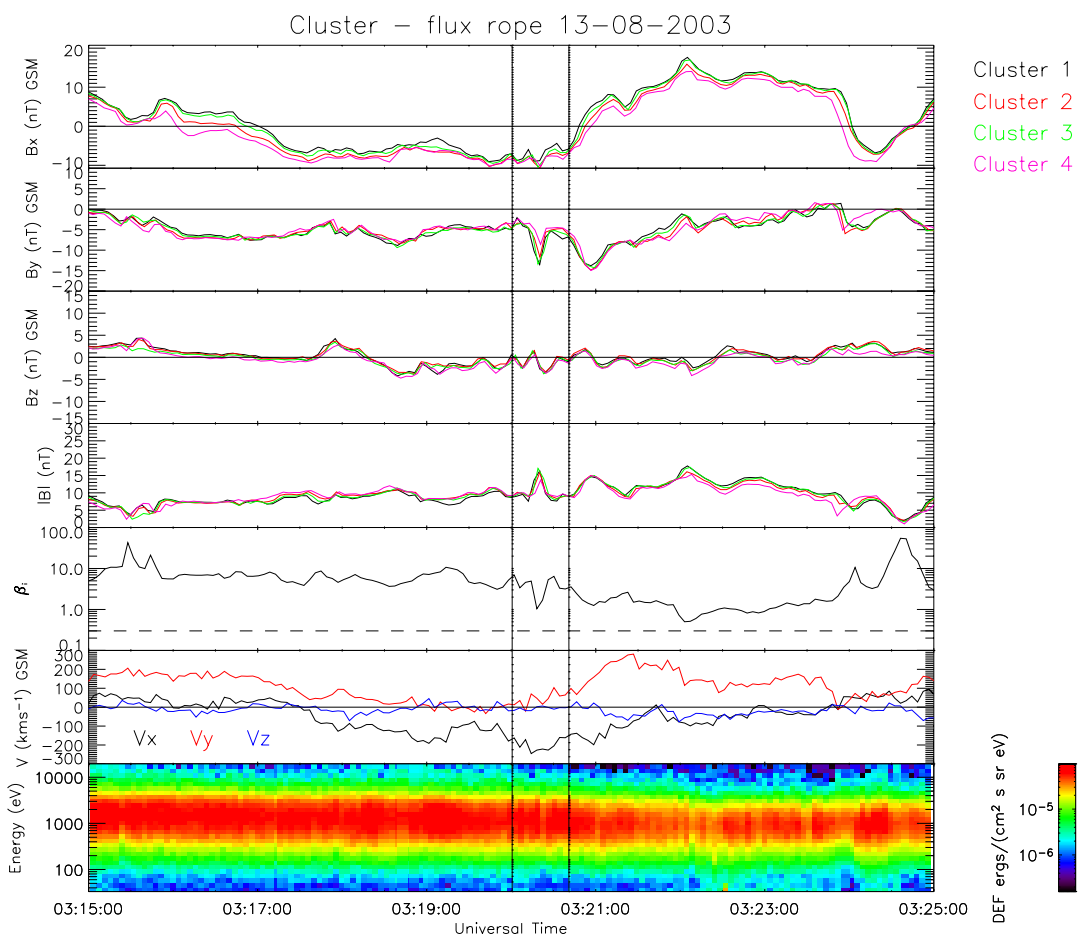

Fig. 10. Cluster observations of 13 August 2003 in the same format as Fig. 3.

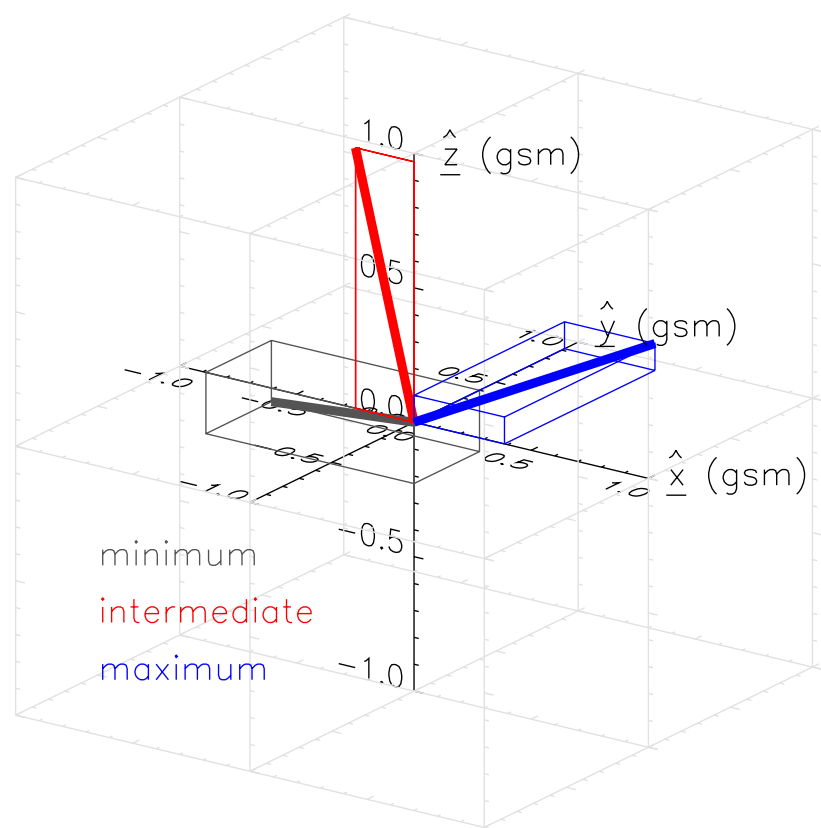

Fig. 11. The variance system in GSM unit space from Cluster 1 for the 13 August event in the same format as Fig. 4. velocity from Cluster1, and the PEACE electron spectrogram in the same format at Fig. 3 .

Again the PEACE electron spectrogram together with $\mathrm{B}_{\mathrm{X}}$ $\sim-7 \mathrm{nT},|\boldsymbol{B}| \sim 7 \mathrm{nT}$ and $\beta_{\mathrm{i}}$ ranging from $\sim 1$ to 10 confirms that the Cluster spacecraft were in the plasma sheet throughout this period. At approximately 03:20:00 UT a large spike in $|\boldsymbol{B}|$ (to $\sim 17 \mathrm{nT}$ ) is concurrent with a small north-thensouth bipolar $\mathrm{B}_{\mathrm{Z}}$ signature. The $\mathrm{AE}$ index for this time indicates that the magnetosphere was in the expansion phase of a large substorm. The polarity of the bipolar signature suggests a flux rope moving tailward. The flux rope is embedded in a fast tailward flow, with $V_{x} \sim-250 \mathrm{kms}^{-1}$. The core field deflection is in (negative) $\mathrm{B}_{\mathrm{y}}$ (from $\sim-3 \mathrm{nT}$ to $\sim-15 \mathrm{nT}$ ), with very little variation in $B_{x}$, indicating that the strong core field was in the-Y-direction.

A minimum variance analysis was carried out on high resolution magnetic field data for this period and Fig. 11 shows the variance directions determined from Cluster 1 with respect to the GSM axes in the same format as Fig. 4. This spacecraft again returned the largest separation of eigenvalues. However, the ratio of eigenvalues for this event is $1: 41.4: 149$, suggesting that the minimum variance direction is determined rather better than the intermediate and maximum variance direction. The minimum variance direction is mainly in the $-\mathrm{X}$-direction. The intermediate variance direction is close to the $+\mathrm{Z}$-direction. In this case it is the maximum variance direction which lies almost along the 


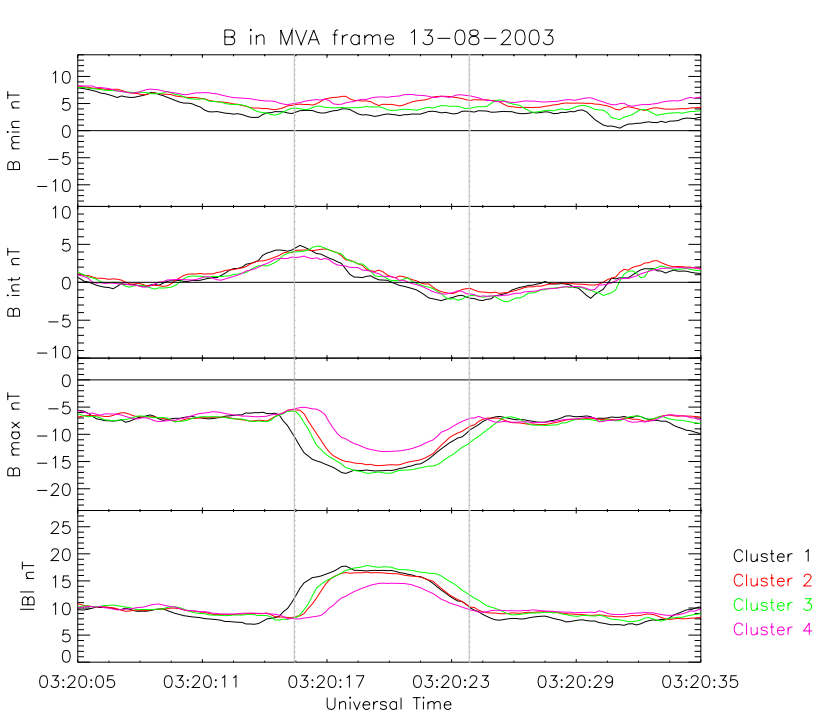

Fig. 12. Magnetic field observations from 13 August event transformed into the variance frame of Cluster 1 in the same format as Fig. 5.

+ Y-direction, most consistent with the direction of the core magnetic field. This flux rope is therefore not-consistent with the expectations of the constant $\alpha$ force-free flux rope discussed in Sect. 2.

Figure 12 shows the magnetic field plotted in the variance frame in the same format as Fig. 5. The small bipolar signature observed in Fig. 10 becomes clear in the intermediate variance direction, while the unipolar core field appears in the maximum variance direction. It is therefore expected that the maximum variance direction should closely correspond to the axis in agreement with the magnetic field observations. The $|\boldsymbol{B}|$ signature suggests a closest approach time of 03:20:20 UT. 4SCT can be used to provide an independent test of the interpretation of this result and was performed on surfaces of constant magnetic flux magnitude. The $\underline{\boldsymbol{V}}_{\mathrm{timg}}$ vectors produced from $4 \mathrm{SCT}$ of inbound $(9 \mathrm{nT})$ and outbound $(10.5 \mathrm{nT})$ surfaces in the variance frame of Cluster 1 can be seen in Fig. 13, in the same format as Fig. 6. In this figure both vectors lie close to the minimum-intermediate plane and thus define a plane whose normal is close to the maximum variance direction, indeed consistent with the axis of this flux rope being directed along the maximum variance direction (see Eq. 3). This result confirms that the variance analysis has successfully determined a good approximation to a "flux rope frame". However, in contrast to the previous case, the maximum variance direction is the appropriate flux rope axis. By finding the appropriate velocity along the minimum variance direction (assumed to be approximately the same direction as the flux rope trajectory) for both inbound $\left(180 \mathrm{kms}^{-1}\right)$ and outbound $\left(220 \mathrm{kms}^{-1}\right)$ vectors and taking an average, a velocity of $200 \mathrm{kms}^{-1}$ is resultant. This

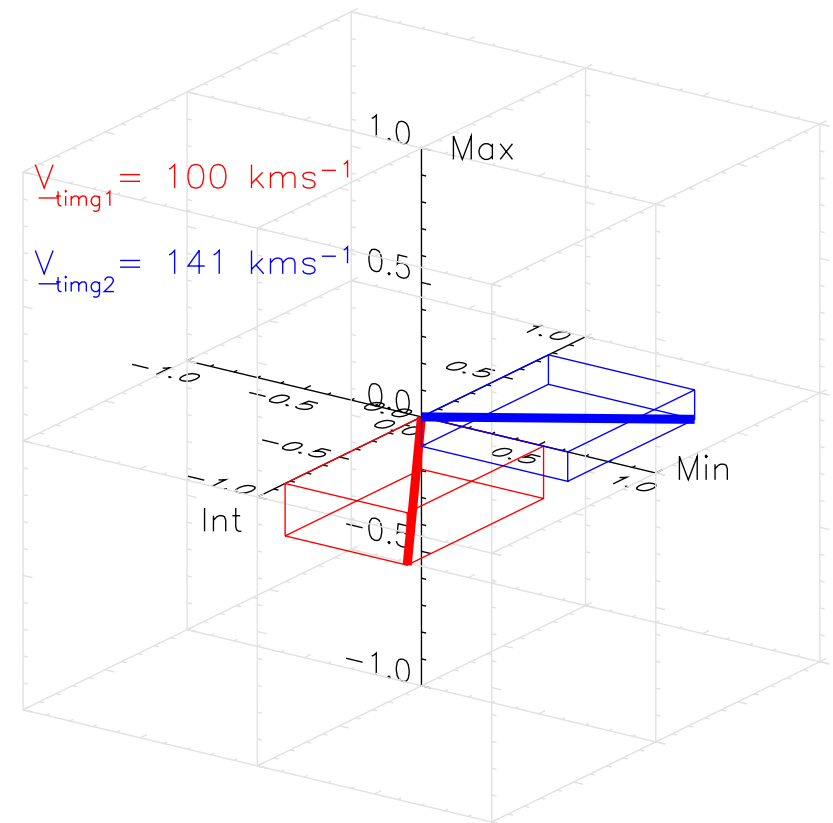

Fig. 13. $\underline{V}_{\text {timg }}$ vectors produced from constant flux surfaces on 13 August event in the unit variance frame in the same format as Fig. 6. The directions "Min", "Int" and "Max" correspond to the minimum, intermediate and maximum variance directions plotted in GSM unit space in Fig. 11.

velocity again corresponds to a size of $\sim 0.3 R_{E}$ (taking the time between the inflection points of $\sim 10 \mathrm{~s}$ ) for this flux rope. Again this is a lower estimate for the size of the structure. The velocity of this flux rope shows that it is travelling tailwards, consistent with the north-then-south polarisation of the bipolar signature. CIS ion velocities rotated into the minimum variance frame (not shown) give a velocity along the minimum variance direction of $\sim 200 \mathrm{kms}^{-1}$ in good agreement with the velocities derived from 4SCT, and along the intermediate variance direction of $\sim 50 \mathrm{kms}^{-1}$ and maximum variance direction of $\sim-50 \mathrm{kms}^{-1}$.

The curlometer technique described above was applied to this period of data and can be seen in Fig. 14, in the same format as Fig. 8. At the beginning of the encounter the magnitude of the perpendicular current (red) is approximately the same as the parallel current (black). In the middle of the flux rope (03:20:20 UT) however, the perpendicular current falls to a level below the parallel current and then climbs again to the level of the parallel current at the end of the encounter. The parallel current stays almost steady throughout the flux rope encounter, with the change in the magnitude of the current being due to the change in the perpendicular component. It can be seen that the current is unidirectional in the minimum (black) and maximum (blue) variance directions and that it undergoes a direction reversal in the intermediate variance direction (red). It would appear therefore that the current circulates around the axis as 


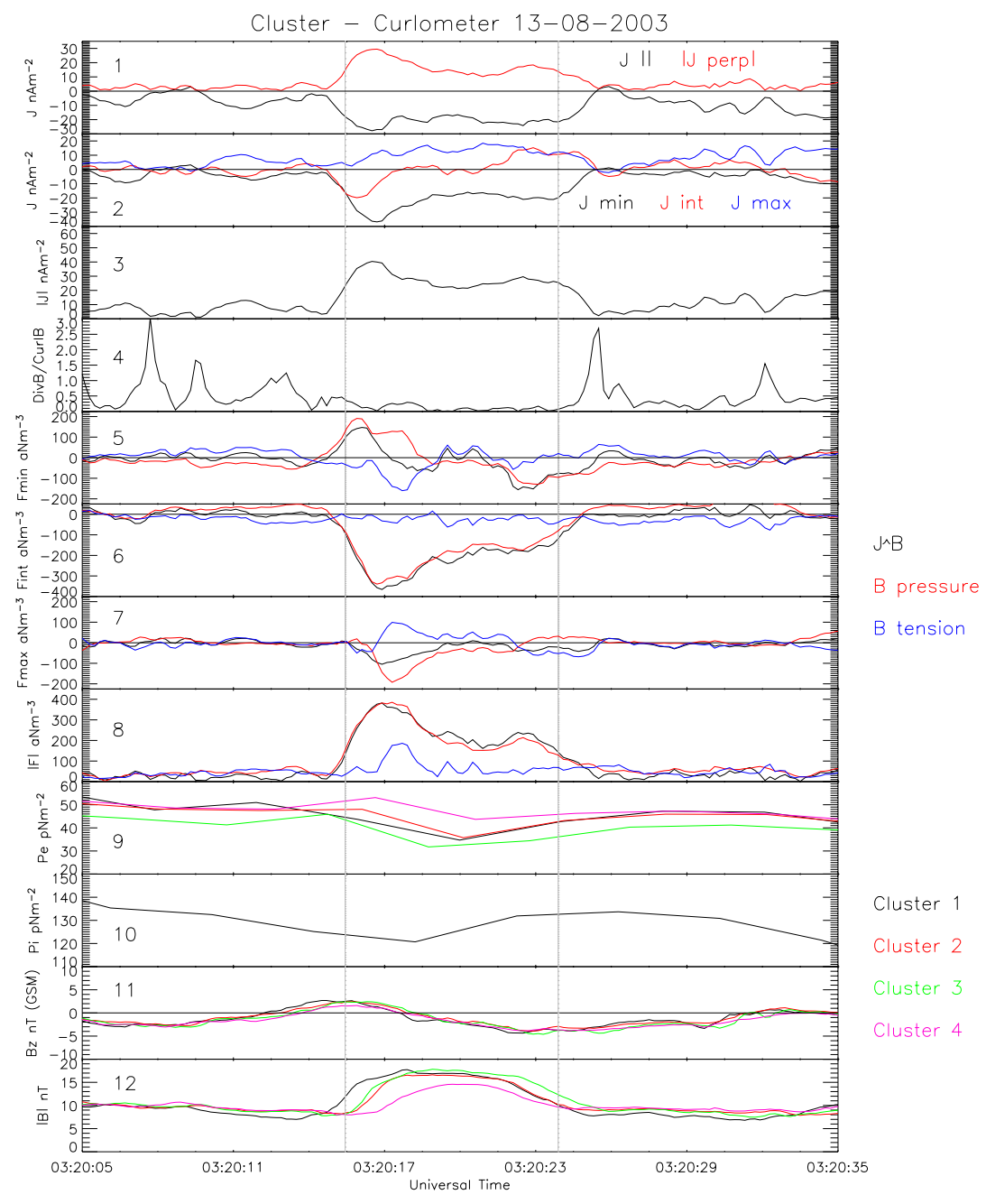

Fig. 14. Curlometer results from 13 August event in the same format as Fig. 8.

well as having an axial component. For this event $\mathrm{E}=0.21$, $\mathrm{P}=0.27$ giving a $\sqrt{E^{2}+P^{2}}$ of 0.34 . The tetrahedron is again a good pseudo-sphere and $\xi$ (Eq. 1) is identified with the relative error in the curlometer current. $\xi$ (Panel 4) is less than 0.3 throughout the flux rope, meaning the quality of the curlometer result should be high. Panels 5 to 12 show the components of the magnetic forces (barycentric magnetic field used) transformed into the variance frame of Cluster 1, their magnitudes, the electron and ion pressure from PEACE and CIS, the $\mathrm{B}_{\mathrm{Z}}$ component and $|\boldsymbol{B}|$. The magnetic forces along the minimum variance direction (assumed to be the trajectory) shown in Panel 5 exhibit direction reversals in both total and magnetic pressure force (positive at 03:20:16 UT, negative at 03:20:23 UT). The direction reversals and the pressure dominance of the magnetic force suggests that the magnetic force is acting to expand the flux rope. A comparison across Panels 5 to 7 shows that most of the magnetic force is in the plane perpendicular to the axis. The domination of the magnetic pressure force over the magnetic tension force over the flux rope agrees with the observed large core field and small bipolar magnetic field. The non-constant $\alpha$ force-free nature of the flux rope is confirmed in Panel 8, showing a large increase in the magnitude of $\boldsymbol{J} \times \boldsymbol{B}$ inside the flux rope. However, this force is again weaker in the centre than in the outer sections. The electron pressure from PEACE (Panel 9) is reduced inside the flux rope as is the ion pressure (Panel 10).

A simple comparison of the change in plasma pressure and magnetic pressure over the first (Cluster 1 ion and magnetic pressure evaluated at 03:20:10 and 03:20:18 UT, Cluster 1 electron pressure evaluated at 03:20:12 and 03:20:20 UT) and second halves of the flux rope (ion and magnetic pressure evaluated at 03:20:18 and 03:20:26 UT, electron pressure evaluated at 03:20:20 and 03:20:28 UT) show that the change in magnetic pressure is larger than the change in plasma pressure by a factor of $\sim 3.1$ and $\sim 3.6$ respectively. It is possible therefore that this flux rope was under a large expansive magnetic pressure dominated total force. 

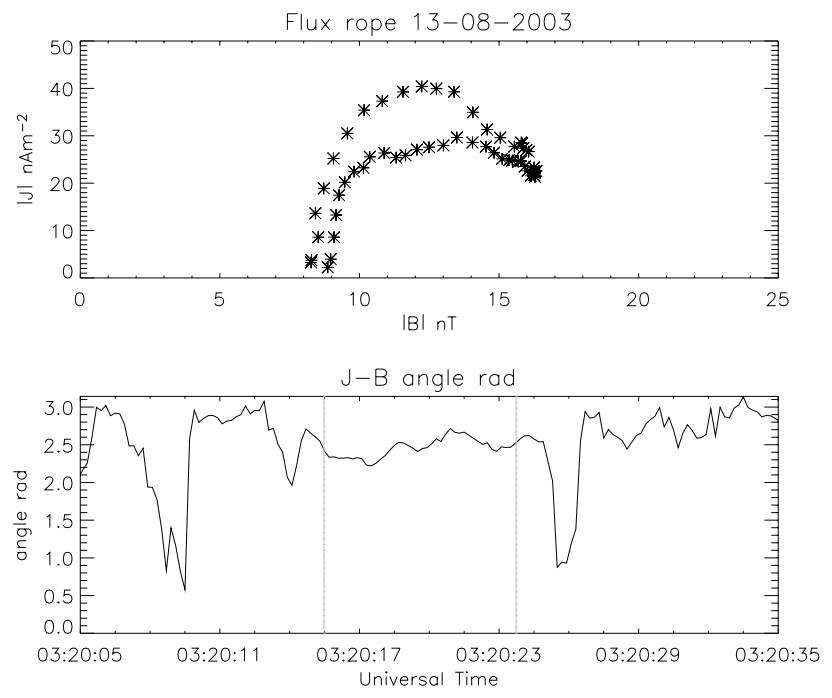

Fig. 15. $|\boldsymbol{J}|$ versus $|\boldsymbol{B}|$ and the angle (in radians) between the magnetic field and current density vectors for the 13 August 2003 flux rope.

Figure 15 shows a scatter plot of the magnitudes of the current density and magnetic field, along with the angle (in radians) between the current density and magnetic field vectors. Again, $|\boldsymbol{J}|$ versus $|\boldsymbol{B}|$ yields no clear proportionality and there exists a quasi-constant but non-zero angle between the current density and magnetic field vectors. These results are inconsistent with the flux rope being in a constant $\alpha$ forcefree configuration.

Again, an independent estimate of the axis direction can be made if the magnetic force is assumed to be only radially acting. A minimum variance analysis carried out on $\boldsymbol{J} \times \boldsymbol{B}$ gives the minimum variance direction to be $(0.514,0.858,-0.056)$ with a minimum-intermediate eigenvalue ratio of 11.9. This makes an angle of $12^{\circ}$ to the maximum variance direction of the magnetic field: $(0.386,0.917,0.100)$. The non-axial nature of the current is confirmed from the maximum variance direction of the current density; $(-0.993,0.063,-0.099)$. This makes an angle of $109^{\circ}$ to the assumed axis.

\section{Discussion}

The event observed by the Cluster spacecraft on the 2 October 2003 was moving Earthward. From a trivial investigation of the magnetic field components, the flux rope axis was not expected to be simply aligned in the \pm Y-direction nor moving solely in the $\pm X$-direction. The evidence for this came from the core field being in the $(+\mathrm{X},-\mathrm{Y},+\mathrm{Z})$-direction. This direction was found to be close to the negative intermediate variance direction of the magnetic field, the direction along which the smallest component of the magnetic force was directed, as well as being the direction along which the majority of the derived current was directed. The current

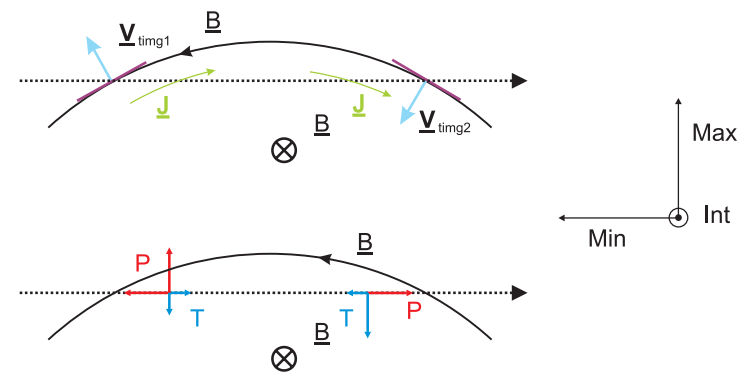

Fig. 16. Schematics of the main results of the 2 October 2003 flux rope. The magnetic field (Fig. 5), 4SCT vectors (Fig. 6) and current (Fig. 8) marked on one schematic, the magnetic pressure (red) and tension (blue, Fig. 8) marked on the other in the variance analysis frame. The relative trajectory of the spacecraft through the flux rope is marked by a dotted line.

was not maximum in the centre of the flux rope. The $\underline{V}_{\text {timg }}$ produced in 4SCT also defined a plane whose normal was close to the intermediate variance direction, which thus can be safely concluded to be the axis of the flux rope in this case. It has been noted in previous studies (Moldwin and Hughes, 1991; Slavin et al., 2003b) that minimum variance analysis does not always satisfactorily define a flux rope frame. The multi-spacecraft timing method employed here is thus a useful test of the derived variance frame. In this case the test verified that the minimum variance analysis technique did indeed provide a good approximation to the "flux rope frame". The duration of the flux rope bipolar signature corresponds to a size of $\sim 0.3 R_{E}$ and a velocity of $\sim 160 \mathrm{kms}^{-1}$, in approximate agreement with CIS velocity moments. The size of the flux rope quoted here is a lower estimate of the diameter, $0.3 R_{E}$ corresponding to the distance between the boundaries of the flux rope at this impact parameter. The larger speed of the outbound $\underline{\boldsymbol{V}}_{\text {timg }}$ vector compared to the inbound vector shows that the constant flux surfaces are either closer together on the tailward side than on the Earthward side of the flux rope, that the flux rope is accelerating or that the flux rope is being compressed during the time that it is observed.

Figure 16 shows a summary of these interpretations in the form of two schematics. The first schematic shows the flux rope with the magnetic field (Fig. 5), 4SCT vectors (Fig. 6) and current (Fig. 8) marked. The magnetic pressure (red) and tension forces (blue, Fig. 8) are marked on the second schematic. Both of these schematics are in the variance analysis frame. The magnetic force along the minimum variance direction exhibits a tripolar signature (Fig. 8). This signature can be thought of as two bipolar signatures, one centred around the flux rope closest approach time and one observed either outside, or on the outer boundary of the flux rope. The magnetic tension and pressure forces in this flux rope appear to be unbalanced (Figs. 8 and 16). Throughout the duration of the first bipolar signature in the force along the minimum variance direction, the magnetic pressure force 


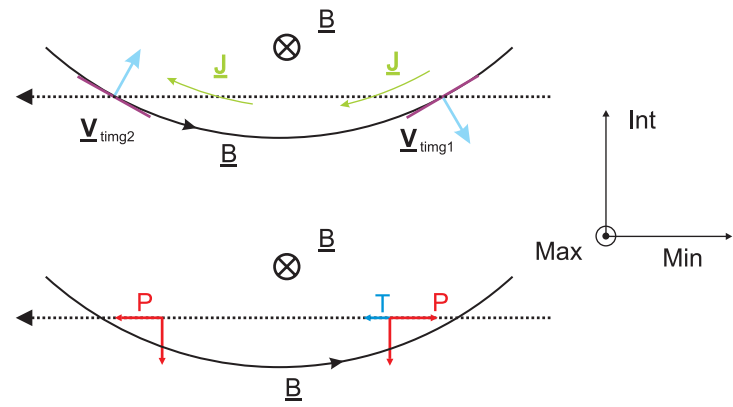

Fig. 17. Schematic of the main results of the 13 August 2003 flux rope in the same format as Fig. 16

is acting away from the flux rope centre (Fig. 16), whilst the magnetic tension force is acting towards the flux rope centre. In the maximum variance direction, the magnetic tension force is generally acting towards the flux rope centre, with the magnetic pressure force acting somewhat away from the flux rope centre (Fig. 16). The resolution of these forces, $\boldsymbol{J} \times \boldsymbol{B}$, is generally acting away from the flux rope centre, i.e. acting to expand the flux rope. The magnetic force along the intermediate variance direction is generally smaller than any other component, showing that the force is mainly acting radially. The magnetic force which comprises the second bipolar signature along the minimum variance direction, the large positive pressure dominated enhancement in $\boldsymbol{J} \times \boldsymbol{B}$ as the spacecraft leaves the flux rope, is most likely acting to push the flux rope Earthward. This could be caused by effects outside the flux rope such as a compression of the flux rope caused by the outflow from a nearby X-line, in agreement with the increased velocity of constant flux surfaces measured on the outbound part of the flux rope (Fig. 6). The magnetic pressure dominance in this flux rope describes a strong core magnetic field and a circulating current. A simple comparison of the changes of plasma and magnetic pressure over the flux rope suggested that the flux rope was experiencing an expansive total force.

The event observed by the Cluster spacecraft on the $13 \mathrm{Au}-$ gust 2003 was moving tailward. The flux rope, expected to be aligned to the \pm Y-direction and travelling in the $\pm \mathrm{X}$ direction from trivial investigations of the magnetic field, was not expected to be in a constant $\alpha$ force-free configuration. The evidence for this came from the observation of a large core field in the -Y direction, this direction being close to the maximum variance direction of the magnetic field and the direction along which the least of the magnetic force was directed. This was confirmed by the $\underline{\boldsymbol{V}}_{\text {timg }}$ produced in $4 \mathrm{SCT}$ which defined a plane whose normal was close to the maximum variance direction, again confirming that the minimum variance analysis returned a good approximation to a "flux rope frame". The flux rope bipolar signature duration corresponded to a size of $0.3 R_{E}$, the flux rope was travelling at $\sim 200 \mathrm{kms}^{-1}$, in agreement with CIS velocity moments. The current was not mainly directed along the maximum variance direction and was therefore not only axial. The current had significant components along the minimum variance direction (trajectory) and maximum variance direction (axis) and showed a reversal in the intermediate variance direction. It therefore appears that the current was circulating in a plane whose normal was the axis.

Figure 17 shows two schematics of the 13 August 2003 flux rope in the same format as Fig. 16. The magnetic force is again unbalanced with the magnetic pressure force along the minimum variance direction being bipolar (Fig. 14), acting away from the flux rope centre (Fig. 17). The magnetic tension force along this direction opposes the magnetic pressure force over the first half of the flux rope, i.e. toward the flux rope centre, falling to zero over the second half of the flux rope. Along the intermediate variance direction, the magnetic pressure is acting away from the flux rope centre, acting to expand the flux rope. The force along the maximum variance direction is small, showing that the force is mainly radial. Thus, the total force is magnetic pressure dominated, and is acting to radially expand the flux rope. This flux rope is dominated by a core magnetic field with a weakly circulating magnetic field, agreeing with the weak bipolar signature in the intermediate variance direction (Fig. 12). The current is mainly circulating around the axis (Fig. 14). The lack of magnetic tension as the spacecraft left the flux rope suggests that the spacecraft flew through a region where the magnetic field lines are weakly curved on the scale of the spacecraft separation. A simple comparison of the plasma and magnetic pressure changes over the flux rope was consistent with the flux rope experiencing a large net outward directed force.

The mechanism for the creation of these structures is important for the study of the break-up of current sheets near substorm onset. The flux ropes reported on here are not force-free, indeed tending to be less force-free in the outer sections of the flux rope than in the centre. The cores of these flux ropes would perhaps be expected to relax in time into the constant $\alpha$ force-free flux rope state, the lowest energy state of a helical magnetic field, as is the case for those seen in the distant tail. If the process responsible for the creation of these flux ropes is MXR and if it is occurring close to the point where the flux ropes are observed, the flux ropes might not have had time to fully relax into this force-free state. However, as the flux in the centre of the flux ropes would have reconnected before that in the outer sections, the central flux would have had more time to begin the evolution towards a force-free configuration. The outer sections would therefore be expected to less force-free than the centre, as observed in both flux ropes reported here.

The lower estimate sizes of these flux ropes are consistent with previous studies of the near-tail plasma sheet. Although Slavin et al. (2003a) estimates flux rope diameters of $\sim 2$ to $5 R_{E}$ at $\mathrm{X} \sim-15$ to $-30 R_{E}$ using Geotail, a number of flux ropes had estimated radii in the region $\sim 0$ to $1 R_{E}$. Due to the small sample of flux ropes reported here, this study is 
unable to determine whether flux ropes at $\mathrm{X} \sim-20 R_{E}$ are generally smaller than those observed out to $\mathrm{X} \sim-30 R_{E}$.

Slavin et al. (2003a) find a large range of orientations of flux ropes; the highly inclined orientation of the 2 October 2003 flux rope reported here may not be particularly unusual. The reason for deviation of the flux ropes' orientation away from the GSM axes remains unclear, but could be due to tilted X-lines or the effects of shear in the plasma sheet flows in which the ropes are embedded.

Eastwood et al. (2005) recently reported on the motion of two X-lines in the tail and an associated flux rope. The flux rope reported by Eastwood et al. (2005) was observed 5 min before the 2 October 2003 flux rope reported in this paper. Eastwood et al. (2005) identifies, using different multi-spacecraft timing methods, a flux rope moving at $\sim 140 \mathrm{kms}^{-1}$ in the direction $(0.778,0.595,0.158)$. This direction is approximately the same as the minimum variance direction reported here and the velocity is approximately the same as reported using 4SCT rotations here. This could suggest that these two flux ropes could be from the same MXR event.

In Slavin et al. (2003b), the current in the presented flux rope is calculated and is found to be not always parallel to the magnetic field. Figure 3 in their paper shows that at some points the magnitude of the current perpendicular to the magnetic field exceeds the magnitude of the current parallel to the magnetic field. This again shows that near-Earth flux ropes seem not to exhibit the characteristics of a constant $\alpha$ class of force-free flux ropes.

Slavin et al. (2003a) find that approximately $60 \%$ of the flux ropes found between $\mathrm{X} \sim-15$ to $-30 R_{E}$ were a good fit to a force-free model and were generally larger than those reported here. One interpretation of the tailward moving flux ropes observed at the distances that Slavin et al. (2003a) investigated is a sample of "older" and larger tailward moving flux ropes than the 13 August event reported in this paper. The fact that not all flux ropes reported in Slavin et al. (2003a) seem to be force-free could be explained if some are evolving away from the form detailed here to a more forcefree configuration.

In Slavin et al. (2003a) the plasma beta is reduced during a flux rope encounter (their Fig. 7). The authors note that without the benefit of high resolution plasma instruments to resolve inside the flux rope, this reduction could simply be caused by an increase in the magnetic field rather than by any reduction in plasma pressure. Figures 8 and 14 of this paper would suggest that the reduction could be due a combination of both factors.

There is a build up of plasma pressure on the Earthward side of the 2 October 2003 Earthward-moving flux rope (not shown). This, along with a large enhancement of current just before the flux rope core (Fig. 8), could be explained if the flux rope was pushing up against oppositely directed terrestrial closed magnetic field lines. There would be a "snow plough" effect as the plasma and magnetic field Earthward of this flux rope was compressed by the flux rope. As the flux rope magnetic field lines and the closed field lines have opposite polarity, re-reconnection could take place, essentially stripping away the outer layers of the flux rope as it travelled further Earthward. If this were the case a small current sheet would be formed with opposite polarity to that inside the flux rope. Indeed, Fig. 8 shows that the current enhancement is mainly in the intermediate variance direction of the magnetic field, i.e. in the axial direction, but directed oppositely to the current inside the flux rope.

Both of the flux ropes reported here are under expansive magnetic forces in their outer sections. These observations could be due to their evolution towards the force-free configuration seen further down the tail after formation in MXR. If this is the case and the 13 August event is a young flux rope, multiple $\mathrm{X}$ point reconnection must have happened shortly Earthward of $\sim 18 R_{E}$ down the tail.

\section{Conclusions}

Few well-formed flux ropes were found in the 2003 Cluster tail season, but two have been investigated in detail in this paper. Neither flux rope was found to be in a magnetic force free configuration, demonstrated by the computation of the $\boldsymbol{J} \times \boldsymbol{B}$ forces inside the flux ropes. The magnetic force was smaller in the centre than in the outer sections of the flux ropes and was generally small along the axis. The magnetic force was magnetic pressure dominated and generally acted away from the flux rope centres, i.e. acting to make the flux ropes radially expand. In one case the axis of the flux rope was in the intermediate variance direction, in the other the axis was in the maximum variance direction. The axis directions were confirmed using multi-spacecraft timings, and were consistent with trivial observations of the magnetic field. The flux ropes were small and slow moving, derived from multi-spacecraft timings, consistent with CIS ion moments. Plasma pressure (both electron and ion) was reduced inside the flux ropes. Both flux ropes were under net expansive total forces, this force imbalance being larger in the 13 August 2003 (tailward moving) flux rope.

These flux ropes have been interpreted as evidence for multiple $\mathrm{X}$-line reconnection in the near-tail close to Cluster apogee $\left(\mathrm{X} \sim-20 R_{E}\right)$. If the flux ropes are newly formed their centres may have had more time to relax towards the force-free state observed further down the tail, consistent with observations of smaller magnetic forces in the centres of the flux ropes. These more distant-tail flux ropes are generally much larger than those observed here, possibly consistent with the expansive magnetic pressure dominance of the $\boldsymbol{J} \times \boldsymbol{B}$ force and overall force imbalance in the 13 August 2003 flux rope. The observation of a tailward moving flux rope suggests that this flux rope was created Earthward of $\mathrm{X} \sim-18 R_{E}$. 
Acknowledgements. The authors wish to thank Y. Bogdanova for the generation of CIS moments. PDH acknowledges UK PPARC studentship funding. IVA was supported by the MSSL PPARCfunded rolling grant and benefited from travel funds associated with an INTAS grant (03-51-3738).

Topical Editor T. Pulkkinen thanks X. H. Deng and another referee for their help in evaluating this paper.

\section{References}

Balogh, A., Carr, C. M., Acuña, M. H., Dunlop, M. W., Beek, T. J., Brown, P., Fornaçon, K.-H., Georgescu, E., Glassmeier, K.-H., Harris, J., Musmann, G., Oddy, T., and Schwingenschuh, K.: The Cluster Magnetic Field Investigation: overview of in-flight performance and initial results, Ann. Geophys., 19, 1207-1217, 2001.

Deng, X. H., Matsumoto, H., Kojima, H., Mukai, T., Anderson, R., Baumjohann, W., and Nakamura, R.: Geotail encounter with reconnection diffusion region in the Earth's magnetotail: Evidence of multiple X lines collisionless reconnection?, J. Geophys. Res., 109, A05 206, doi:10.1029/2003JA010031, 2004.

Dunlop, M. W., Balogh, A., Glassmeier, K.-H., and Robert, P.: Four-point Cluster application of magnetic field analysis tools: The Curlometer, J. Geophys. Res., 107, 1384-1397, 2002.

Eastwood, J. P., Balogh, A., Dunlop, M. W., and Smith, C. W.: Cluster observations of the heliospheric current sheet and an associated magnetic flux rope and comparisons with ACE, J. Geophys. Res., 107, 1365-1373, 2002.

Eastwood, J. P., Sibeck, D. G., Slavin, J. A., Goldstein, M. L., Lavraud, B., Sitnov, M., Imber, S., Balogh, A., Lucek, E. A., and Dandouras, I.: Observations of multiple X-line structure in the Earth's magnetotail current sheet: A Cluster case study, Geophys. Res. Lett., 32, L11 105, 2005.

Elphic, R. C., Cattell, C. A., Takahashi, K., Bame, S. J., and Russell, C. T.: ISEE-1 and 2 observations of magnetic flux ropes in the magnetotail: FTEs in the plasma sheet?, Geophys. Res. Lett., 13, 648-651, 1986.

Hughes, W. J. and Sibeck, D. G.: On the 3-dimensional structure of plasmoids, Geophys. Res. Lett., 14, 636-639, 1987.

Johnstone, A. D., Alsop, C., Burdge, S., Carter, P. J., Coates, A. J., Coker, A. J., Fazakerley, A. N., Grande, M., Gowen, R. A., Gurgiolo, C., Hancock, B. K., Narheim, B., Preece, A., Sheather, P. H., Winningham, J. D., and Woodcliffe, R. D.: PEACE, A Plasma Electron and Current Experiment, Space Sci. Rev., 79, 351-398, 1997.

Lepping, R. P., Jones, J. A., and Burlaga, L. F.: Magnetic Field Structure of Interplanetary Magnetic Clouds at 1 AU, J. Geophys. Res., 95, 11 957-11 965, 1990.

Moldwin, M. B. and Hughes, W. J.: Plasmoids as Magnetic Flux Ropes, J. Geophys. Res., 96, 14 051-14 064, 1991.

Rème, H., Aoustin, C., Bosqued, J. M., Dandouras, I., Lavraud, B., Sauvaud, J. A., Barthe, A., Bouyssou, J., Camus, T., CoeurJoly, O., Cros, A., Cuvilo, J., Ducay, F., Garbarowitz, Y., Medale, J. L., Penou, E., Perrier, H., Romefort, D., Rouzaud, J., Vallat, C., Alcaydé, D., Jacquey, C., Mazelle, C., d'Uston, C., Mobius, E., Kistler, L. M., Crocker, K., Granoff, M., Mouikis, C., Popecki, M., Vosbury, M., Klecker, B., Hovestadt, D., Kucharek, H., Kuenneth, E., Paschmann, G., Scholer, M., Sckopke, N., Seidenschwang, E., Carlson, C. W., Curtis, D. W., Ingraham, C.,
Lin, R. P., McFadden, J. P., Parks, G. K., Phan, T., Formisano, V., Amata, E., Bavassano-Cattaneo, M. B., Baldetti, P., Bruno, R., Chionchio, G., Lellis, A. D., Marcucci, M. F., Pallocchia, G., Korth, A., Daly, P. W., Graeve, B., Rosenbauer, H., Vasyliunas, V., McCarthy, M., Wilber, M., Eliasson, L., Lundin, R., Olsen, S., Shelley, E. G., Fuselier, S., Ghielmetti, A. G., Lennartsson, W., Escoubet, C. P., Balsiger, H., Friedel, R., Cao, J.-B., Kovrazhkin, R. A., Papamastorakis, I., Pellat, R., Scudder, J., and Sonnerup, B.: First multispacecraft ion measurements in and near the Earth's magnetosphere with the identical Cluster ion spectrometry (CIS) experiment, Ann. Geophys., 19, 1303-1354, 2001.

Robert, P., Dunlop, M. W., Roux, A., and Chanteur, G.: Accuracy of Current density Determination, in: Analysis Methods for MultiSpacecraft Data, edited by: Pashmann, G. and Daly, P. W., Int, Space Sci, Inst., Bern, 395-418, 1998.

Schindler, K.: A Theory of the Substorm Mechanism, J. Geophys. Res., 79, 2803-2810, 1974.

Slavin, J. A., Owen, C. J., Kuznetsova, M. M., and Hesse, M.: ISEE 3 observations of plasmoids with flux rope magnetic topologies, Geophys. Res. Lett., 22, 2061-2064, 1995.

Slavin, J. A., Lepping, R. P., Gjerloev, J., Fairfield, D. H., Hesse, M., Owen, C. J., Moldwin, M. B., Nagai, T., Ieda, A., and Mukai, T.: Geotail observations of magnetic flux ropes in the plasma sheet, J. Geophys. Res., 108, 1015-1032, 2003 a.

Slavin, J. A., Lepping, R. P., Gjerloev, J., Goldstein, M. L., Fairfield, D. H., Acuña, M. H., Balogh, A., Dunlop, M., Kivelson, M. G., Khurana, K., Fazakerley, A., Owen, C. J., Rème, H., and Bosqued, J. M.: Cluster electric current density measurements within a magnetic flux rope in the plasma sheet, Geophys. Res. Lett., 30, 1362-1365, 2003b.

Slavin, J. A., Transkanen, E. I., Hesse, M., Owen, C. J., Dunlop, M. W., Imber, S., Lucek, E. A., Balogh, A., and Glassmeier, K.-H.: Cluster observations of traveling compression regions in the near-tail, J. Geophys. Res., 110, A06207, doi:10.1029/2004JA010878, 2005.

Sonnerup, B. U. O. and Cahill, L. J.: Magnetopause structure and attitude from Explorer 12 observations, J. Geophys. Res., 72, 171-183, 1967.

Xiao, C. J., Pu, Z. Y., Ma, Z. W., Fu, S. Y., Huang, Z. Y., and Zong, Q. G.: Inferring of flux rope orientation with the minimum variance analysis technique, J. Geophys. Res., 109, A11218, doi:10.1029/2004JA010594, 2004.

Zong, Q.-G., Fritz, T. A., Pu, Z. Y., Fu, S. Y., Baker, D. N., Zhang, H., Lui, A. T., Vogiatzis, I., Glassmeier, K.-H., Korth, A., Daly, P. W., Balogh, A., and Rème, H.: Cluster observations of earthward flowing plasmoid in the tail, Geophys. Res. Lett., 31, L18 803, doi:10.1029/2004GL020692 , 2004. 